\title{
Some New Characterizations of Intrinsic Transversality in Hilbert Spaces
}

\section{Nguyen Hieu Thao ${ }^{1,2}$ (D) Hoa T. Bui ${ }^{3} \cdot$ Nguyen Duy Cuong $^{4,5} \cdot$ Michel Verhaegen $^{1}$}

Received: 31 December 2018 / Accepted: 16 January 2020 / Published online: 12 February 2020

(C) The Author(s) 2020

\begin{abstract}
Motivated by a number of questions concerning transversality-type properties of pairs of sets recently raised by Ioffe and Kruger, this paper reports several new characterizations of the intrinsic transversality property in Hilbert spaces. New results in terms of normal vectors clarify the picture of intrinsic transversality, its variants and sufficient conditions for subtransversality, and unify several of them. For the first time, intrinsic transversality is characterized by an equivalent condition which does not involve normal vectors. This characterization offers another perspective on intrinsic transversality. As a consequence, the obtained results allow us to answer a number of important questions about transversalitytype properties.
\end{abstract}

Keywords Transversality $\cdot$ Subtransversality $\cdot$ Intrinsic transversality $\cdot$ Normal cone Relative normal cone

Mathematics Subject Classification (2010) Primary 49J53 - 65K10; Secondary 49K40 . 49M05 - 49M37 - 65K05 - 90C30

\section{Introduction}

Transversality and subtransversality are the two important properties of collections of sets which reflect the mutual arrangement of the sets around the reference point in normed

Dedicated to Professor Alexander Kruger on the occasion of his 65th birthday

NHT and MV are supported by the European Research Council under the European Union's Seventh Framework Programme (FP7/2007-2013)/ERC grant agreement No. 339681. HTB and NDC are supported by the Australian Research Council, project DP160100854, an Australian Government Research Training Program Fee Off-Set Scholarship, and a CIAO PhD Research Scholarship through Federation University Australia. HTB is also supported by the Australian Research Council through grant IC180100030.

Nguyen Hieu Thao

h.t.nguyen-3@tudelft.nl; nhthao@ctu.edu.vn

Extended author information available on the last page of the article. 
spaces. These properties are widely known as constraint qualification conditions in optimization and variational analysis for formulating optimality conditions $[22,45,47,53]$ and calculus rules for subdifferentials, normal cones and coderivatives [19-21, 23, 33, 34, 45, 47], and as key ingredients for establishing sufficient and/or necessary conditions for linear convergence of computational algorithms [3, 14, 16, 36, 41, 42, 44, 50, 52]. We refer the reader to the papers [26-28, 33-37, 39] by Kruger and his collaborators for a variety of their sufficient and/or necessary conditions in both primal and dual spaces.

Transversality is strictly stronger than subtransversality. It is sufficient for many applications where the latter is not, for example, in proving linear convergence of the alternating projection method for solving nonconvex feasibility problems [41, 42], or in establishing error bounds for the Douglas-Rachford algorithm [16, 50] and its modified variants [52]. However, transversality is too restrictive for many applications, and there have been a number of attempts to identify weaker properties, still sufficient for such applications. Of course, one cannot expect a universal transversality-type property that works well for all applications. When formulating necessary optimality conditions for optimization problems in terms of abstract Lagrange multipliers and establishing intersection rules for tangent cones in Banach spaces, Bivas et al. [7] recently introduced a property called tangential transversality, which is a primal space property lying between transversality and subtransversality, but there is no evidence that this new property is actually different from both.

When establishing linear convergence criteria of the alternating projection algorithm for solving nonconvex feasibility problems, a series of meaningful transversality-type properties have been introduced and analyzed in the literature: affine-hull transversality [50], inherent transversality [6], separable intersection property [48] and intrinsic transversality [13]. In contrast to tangential transversality, the latter ones are dual space properties since they are defined in terms of normal vectors. Unlike the transversality property, all the above transversality-type properties are not dependent on the underlying space, that is, if a property is satisfied in an ambient space $X$, then it is also satisfied in any ambient space containing $X$. Recall that in Euclidean spaces, a pair of closed sets $\{A, B\}$ is transversal at a common point $\bar{x}$ if and only if

$$
\bar{N}_{A}(\bar{x}) \cap\left(-\bar{N}_{B}(\bar{x})\right)=\{0\},
$$

where $\bar{N}_{A}(\bar{x})$ stands for the limiting normal cone to $A$ at $\bar{x}$, see Eq. 3 for the definition. This characterization reveals that transversality is a property that involves all the limiting normals to the sets at the reference point. This fundamentally explains why the property is not invariant with respect to the ambient space and becomes too restrictive for many applications. Indeed, the hidden idea leading to the introduction of the aforementioned transversality-type properties in the context of nonconvex alternating projection follows from the observation that not all such normal vectors are relevant for analyzing convergence of the algorithm. The affine-hull transversality is the transversality but considered only in the affine hull $L$ of the two sets, that is, the pair of translated sets $\{A-\bar{x}, B-\bar{x}\}$ is transversal at 0 in the ambient space $L-\bar{x}$. As a consequence, the analysis of this property is straightforwardly obtained from that of transversality [50]. The key feature of the inherent transversality ${ }^{1}$ [6] is the use of restricted normal cones in place of the conventional limiting normal cones in characterization Eq. 1 of transversality in Euclidean spaces. As a result, the analysis of this property is

\footnotetext{
${ }^{1}$ The property originated in [6, Theorem 2.13] without a name, then was refined and termed as inherent transversality in Definition 4.4 of the preprint "Drusvyatskiy, D., Ioffe, A.D., Lewis, A.S.: Alternating projections and coupling slope. Preprint, arXiv:1401.7569, 1-17 (2014)".
} 
reduced to the calculus of the restricted normal vectors as established in [6]. The separable intersection property [48, Definition 1] was motivated by nonconvex alternating projection and ultimately designed for this algorithm. The intrinsic transversality was also introduced in the context of nonconvex alternating projection in Euclidean spaces [13], it turns out to be an important property itself in variational analysis as demonstrated by Ioffe [23, Section 9.2] and [22] and Kruger [32]. On the one hand, a variety of characterizations of intrinsic transversality in various settings (Euclidean, Hilbert, Asplund, Banach and normed linear spaces) have been established by a number of researchers [13, 22, 23, 32, 36, 40, 48]. On the other hand, there are still a number of important questions about this property, for example, the ones raised by Kruger [32, page 140] or the challenge by Ioffe about primal counterparts of intrinsic transversality [22, Remark 6.1]. It is known that for pairs of closed and convex sets, subtransversality admits an equivalent characterization in terms of normal vectors, and the latter is equivalent to intrinsic transversality in the Euclidean space setting [32]. Another interesting question is whether this equivalence is also valid in the nonconvex setting.

Motivated mainly by the above research questions, this paper is devoted to investigating further characterizations of intrinsic transversality in connection with other transversalitytype properties. Apart from the appeal to address the aforementioned questions, this work was also motivated by the potential for meaningful applications of these properties, for example, in establishing convergence criteria for more delicate projection algorithms (rather than alternating projection) and in formulating calculus rules for relative limiting normal cones (see Definition 5).

The organization and contribution of the paper is as follows. New results in terms of elements of normal cones are presented in Section 2 with the key quantitative estimate formulated in Lemma 1. Theorem 1 establishes the equivalence of intrinsic transversality, weak intrinsic transversality [32] and the sufficient condition for subtransversality formulated in [35, Theorem 2]. This result significantly clarifies the picture of transversality-type properties and unifies several of them. As by-products, we address several important questions concerning these properties in the Hilbert space setting, see Questions 1-3. In Section 3, for the first time, intrinsic transversality is characterized by an equivalent property which does not involve normal vectors, see Theorem 2. This result, which was motivated by a question (see Question 4) raised by Ioffe [22, Remark 6.1], opens a new perspective on intrinsic transversality. Intrinsic transversality in Euclidean spaces is studied in Section 4. Lemma 3 establishes a geometric counterpart of the analytic condition under which the complete quantitative results of Theorem 1(ii) are obtained. Theorem 3 gives a new characterization of intrinsic transversality, which refines the corresponding result of [32, Corollary 3]. Theorem 4 yields further insight on the quantitative results established in Section 2 when specialized to the Euclidean space setting. As by-products, we address a couple of interesting questions concerning intrinsic transversality in Euclidean spaces raised by Kruger [32, page 140], see Questions 5 and 6.

Our basic notation is standard; cf. [12, 45, 51]. The setting throughout the current paper is a Hilbert space $X$. The open unit ball in $X$ is denoted by $\mathbb{B}$, and $\mathbb{B}_{\delta}(x)$ (respectively, $\overline{\mathbb{B}}_{\delta}(x)$ ) stands for the open (respectively, closed) ball with center $x$ and radius $\delta>0$. The distance from a point $x \in X$ to a set $\Omega \subset X$ is defined by $\operatorname{dist}(x, \Omega):=\inf _{\omega \in \Omega}\|x-\omega\|$, and we use the convention $\operatorname{dist}(x, \Omega)=+\infty$ when $\Omega=\emptyset$. The set-valued mapping

$$
P_{\Omega}: X \rightrightarrows X: x \mapsto\{\omega \in \Omega \mid\|x-\omega\|=\operatorname{dist}(x, \Omega)\}
$$

is the projector on $\Omega$. An element $\omega \in P_{\Omega}(x)$ is called a projection. Note that the projector is not, in general, single-valued and can have empty values. The single-valuedness of $P_{\Omega}$ everywhere in fact defines the Chebyshev property of $\Omega$. Every nonempty closed convex set 
in a Hilbert space is Chebyshev. The inverse of the projector, $P_{\Omega}^{-1}$, is defined by

$$
P_{\Omega}^{-1}(\omega):=\left\{x \in X \mid \omega \in P_{\Omega}(x)\right\} \quad \forall \omega \in \Omega .
$$

The proximal normal cone to $\Omega$ at a point $\bar{x} \in \Omega$ is defined by

$$
N_{\Omega}^{p}(\bar{x}):=\operatorname{cone}\left(P_{\Omega}^{-1}(\bar{x})-\bar{x}\right),
$$

which is a convex cone. Here cone(.) denotes the smallest cone containing the set in the brackets.

The Fréchet normal cone to $\Omega$ at $\bar{x}$ is defined by (cf. [25])

$$
N_{\Omega}(\bar{x}):=\left\{v \in X \mid \limsup _{\substack{\Omega \\ x \rightarrow \bar{x}, x \neq \bar{x}}} \frac{\langle v, x-\bar{x}\rangle}{\|x-\bar{x}\|} \leq 0\right\},
$$

which is a nonempty closed convex cone. Here $x \stackrel{\Omega}{\rightarrow} \bar{x}$ means $x \rightarrow \bar{x}$ and $x \in \Omega$.

The limiting normal cone to $\Omega$ at $\bar{x}$ is defined by

$$
\bar{N}_{\Omega}(\bar{x}):=\underset{\substack{\operatorname{Limsup} \\ x \rightarrow \bar{x}}}{ } N_{\Omega}(x):=\left\{v=\lim _{k \rightarrow \infty} v_{k} \mid v_{k} \in N_{\Omega}\left(x_{k}\right), x_{k} \in \Omega, x_{k} \rightarrow \bar{x}\right\} .
$$

In the above definition, the Fréchet normal cones can equivalently be replaced by the proximal ones. It holds: $N_{\Omega}^{p}(\bar{x}) \subset N_{\Omega}(\bar{x}) \subset \bar{N}_{\Omega}(\bar{x})$, and if $\Omega$ is closed and $\operatorname{dim} X<\infty$, then $\bar{N}_{\Omega}(\bar{x}) \neq\{0\}$ if and only if $\bar{x} \in$ bd $(\Omega)$, where bd $(\Omega)$ denotes the boundary of $\Omega$. By convention, we set $N_{\Omega}^{p}(\bar{x})=N_{\Omega}(\bar{x})=\bar{N}_{\Omega}(\bar{x}):=\emptyset$ whenever $\bar{x} \notin \Omega$. If $\Omega$ is a convex set, then all the above normal cones coincide and reduce to the one in the sense of convex analysis (e.g., [9, Proposition 2.4.4], [25, Proposition 1.19]):

$$
N_{\Omega}^{p}(\bar{x})=N_{\Omega}(\bar{x})=\bar{N}_{\Omega}(\bar{x})=\{v \in X \mid\langle v, x-\bar{x}\rangle \leq 0 \text { for all } x \in \Omega\} .
$$

\section{Subtransversality, Transversality and Intrinsic Transversality}

The following definition recalls probably the most widely known regularity properties of pairs of sets.

Definition 1 (subtransversality and transversality) Let $\{A, B\}$ be a pair of sets and $\bar{x} \in$ $A \cap B$.

(i) $\{A, B\}$ is subtransversal at $\bar{x}$ if there exist numbers $\alpha \in] 0,1[$ and $\delta>0$ such that

$$
\alpha \operatorname{dist}(x, A \cap B) \leq \max \{\operatorname{dist}(x, A), \operatorname{dist}(x, B)\} \quad \forall x \in \mathbb{B}_{\delta}(\bar{x}) .
$$

(ii) $\{A, B\}$ is transversal at $\bar{x}$ if there exist numbers $\alpha \in] 0,1[$ and $\delta>0$ such that

$$
\begin{aligned}
& \alpha \operatorname{dist}\left(x,\left(A-x_{1}\right) \cap\left(B-x_{2}\right)\right) \leq \max \left\{\operatorname{dist}\left(x, A-x_{1}\right), \operatorname{dist}\left(x, B-x_{2}\right)\right\} \\
& \forall x \in \mathbb{B}_{\delta}(\bar{x}), \forall x_{1}, x_{2} \in \delta \mathbb{B} .
\end{aligned}
$$

The exact upper bound of all $\alpha \in$ ]0, 1 [ such that condition Eq. 4 (respectively, Eq. 5) is satisfied for some $\delta>0$ is denoted by $\operatorname{str}[A, B](\bar{x})$ (respectively, $\operatorname{tr}[A, B](\bar{x})$ ) with the convention that the supremum over the empty set equals 0 . It is clear that Eq. 5 implies Eq. 4 by setting $x_{1}=x_{2}=0$ in Eq. 5 . Hence, transversality is stronger than subtransversality, and it always holds that $\operatorname{tr}[A, B](\bar{x}) \leq \operatorname{str}[A, B](\bar{x})$. 
Remark 1 (i) (subtransversality) The subtransversality property can be traced back to at least the early 80 's thanks to Dolecki under the name decisive separation $[10,11]$ where it was known as a sufficient (and also necessary in the convex setting) condition for the tangent cone of the intersection of a pair of sets at a reference point being equal to the intersection of the two tangent cones of the sets at that point [11, Propositions 5.3 and 5.4]. In the surveys [18, 19], Ioffe used the property (without a name) as a qualification condition for establishing calculus rules for normal cones and subdifferentials. Subtransversality was studied by Bauschke and Borwein [2] under the name linear regularity as a sufficient condition for linear convergence of the alternating projection algorithm for solving convex feasibility problems in Hilbert spaces, and became widely known thanks to this important application. Their results were extended to the cyclic projection algorithm for solving feasibility problems involving a finite number of convex sets [3]. The term linear regularity was widely adapted in the community of variational analysis and optimization for several decades, for example, Bakan et al. [1], Bauschke et al. [4, 5], Li et al. [43], Ng and Zang [46], Zheng and Ng [54], Kruger and his collaborators [26-28, 37-39]. Ngai and Théra [47] referred to this property as metric inequality and used it to establish calculus rules for the limiting Fréchet subdifferential. Penot [49] referred to the property as linear coherence and applied it in establishing calculus rules for the viscosity Fréchet and viscosity Hadamard subdifferentials. The name (sub)transversality was coined by Ioffe in the 2016 survey [20, Definition 6.14]. In his 2017 book [22, page 301] he explained that "Regularity is a property of a single object while transversality relates to the interaction of two or more independent objects". In spite of the relatively long history with many important features of subtransversality, for example, those in connection with metric subregularity, error bounds, weak sharp minima, growth conditions and conditions involving primal and dual space slopes, useful applications of the property keep being discovered. For example, Luke et al. [44, Theorem 8] recently proved that subtransversality is not only sufficient but also necessary for linear convergence of convex alternating projection. This complements the aforementioned result by Bauschke and Borwein [2] obtained 25 years earlier. Luke et al. [44, Section 4] also reveal that the property has been imposed either explicitly or implicitly in all existing linear convergence criteria for nonconvex alternating projection, and hence conjecture that subtransversality is a necessary condition for linear convergence of the algorithm also in the nonconvex setting.

(ii) (transversality) The origin of the concept of transversality can be traced back to at least the 19th century (cf. $[15,17]$ ) in differential geometry which deals of course with smooth manifolds, where transversality of a pair of smooth manifolds $\{A, B\}$ at a common point $\bar{x}$ can also be characterized by condition Eq. $1 .^{2}$ The property is known as a sufficient condition for the intersection $A \cap B$ to be also a smooth manifold around $\bar{x}$. To the best of our awareness, transversality of pairs (collections) of general sets in normed linear spaces was first investigated by Kruger in a systematic picture of mutual arrangement properties of sets. The property has been known under quite a number of other names including regularity, strong regularity, property $(U R)_{S}$, uniform regularity, strong metric inequality [26-28, 37] and linear regular intersection [41]. Plenty of primal and dual space characterizations of transversality as well as its

\footnotetext{
${ }^{2}$ In this setting, the normal cones appearing in Eq. 1 are the normal spaces (i.e., orthogonal complements to the tangent spaces) to the manifolds at $\bar{x}$. The minus sign in Eq. 1 can be omitted.
} 
close connections to important concepts in optimization and variational analysis such as metric regularity, (extended) extremal principles, open mapping theorems and other types of mutual arrangement properties of collections of sets have been established and extended to more general nonlinear settings in a series of papers by Kruger and his collaborators [24, 26-31, 38, 39]. Apart from classical applications of the property, for example, as constraint qualification conditions for establishing calculus rules for the limiting normal cones [45, page 265] and coderivatives (in connection with metric regularity, the counterpart of transversality in terms of set-valued mappings) [12, 51], important applications have also emerged in the field of numerical analysis. Lewis et al. [41, 42] applied the property to establish the first linear convergence criteria for nonconvex alternating and averaged projection. Transversality was also used to prove linear convergence of the Douglas-Rachford algorithm [16, 50] and its relaxations [52].

We refer the reader to the recent surveys by Kruger et al. [35, 36] for a more comprehensive discussion about the two properties in Definition 1.

A number of characterizations of transversality in terms of normal vectors, especially in the Euclidean space setting, have been established [26-28, 36, 37, 39, 41] and applied, for example, $[41,45,50,52]$. The situation is very much different for subtransversality. For collections of closed and convex sets, the following characterization of subtransversality is due to Kruger.

Proposition 1 (normal-vector-based characterization of subtransversality with convexity) $[32 \text {, Theorem } 3]^{3}$ A pair of closed and convex sets $\{A, B\}$ is subtransversal at a point $\bar{x} \in A \cap B$ if and only if there exist numbers $\alpha \in] 0,1\left[\right.$ and $\delta>0$ such that $\left\|v_{1}+v_{2}\right\|>\alpha$ for all $a \in(A \backslash B) \cap \mathbb{B}_{\delta}(\bar{x}), b \in(B \backslash A) \cap \mathbb{B}_{\delta}(\bar{x}), x \in \mathbb{B}_{\delta}(\bar{x})$ with $\|x-a\|=\|x-b\|$ and $v_{1}, v_{2} \in X$ satisfying

$$
\begin{gathered}
\operatorname{dist}\left(v_{1}, N_{A}(a)\right)<\delta, \operatorname{dist}\left(v_{2}, N_{B}(b)\right)<\delta, \\
\left\|v_{1}\right\|+\left\|v_{2}\right\|=1,\left\langle v_{1}, x-a\right\rangle=\left\|v_{1}\right\|\|x-a\|,\left\langle v_{2}, x-b\right\rangle=\left\|v_{2}\right\|\|x-b\| .
\end{gathered}
$$

Let $\operatorname{itr}_{c}[A, B](\bar{x})$ denote the exact upper bound of all $\left.\alpha \in\right] 0,1[$ such that the conditions in Proposition 1 (equivalent to subtransversality in the convex setting) are satisfied for some $\delta>0$, with the convention that the supremum over the empty set equals 0 . This quantity is well defined regardless of the convexity of the sets, and the strict inequality $\operatorname{itr}_{c}[A, B](\bar{x})>$ 0 characterizes a certain transversality-type property in not necessarily convex settings. The constant $\operatorname{itr}_{c}[A, B](\bar{x})$ is going to play a central role in the subsequent analysis of this paper.

In the nonconvex setting, the first sufficient condition for subtransversality in terms of normal vectors was formulated in [38, Theorem 4.1] following the routine of deducing metric subregularity characterizations of set-valued mappings in [29]. The result was then refined successively in [36, Theorem 4(ii)], [35, Theorem 2] and finally in [32] in the following form.

Proposition 2 (sufficient condition for subtransversality) [32, combination of Definition 2 and Corollary 2$]^{4}$ A pair of closed sets $\{A, B\}$ is subtransversal at a point $\bar{x} \in A \cap B$

\footnotetext{
${ }^{3}$ The result is valid in Banach spaces.

${ }^{4}$ The result is valid in Asplund spaces.
} 
if there exist numbers $\alpha \in] 0,1\left[\right.$ and $\delta>0$ such that, for all $a \in(A \backslash B) \cap \mathbb{B}_{\delta}(\bar{x}), b \in$ $(B \backslash A) \cap \mathbb{B}_{\delta}(\bar{x})$ and $x \in \mathbb{B}_{\delta}(\bar{x})$ with $\|x-a\|=\|x-b\|$, one has $\left\|v_{1}+v_{2}\right\|>\alpha$ for some $\varepsilon>0$ and all $a^{\prime} \in A \cap \mathbb{B}_{\varepsilon}(a), b^{\prime} \in B \cap \mathbb{B}_{\varepsilon}(b), x_{1}^{\prime} \in \mathbb{B}_{\varepsilon}(a), x_{2}^{\prime} \in \mathbb{B}_{\varepsilon}(b)$ with $\left\|x-x_{1}^{\prime}\right\|=\left\|x-x_{2}^{\prime}\right\|$, and $v_{1}, v_{2} \in X$ satisfying

$$
\begin{gathered}
\operatorname{dist}\left(v_{1}, N_{A}\left(a^{\prime}\right)\right)<\delta, \operatorname{dist}\left(v_{2}, N_{B}\left(b^{\prime}\right)\right)<\delta \\
\left\|v_{1}\right\|+\left\|v_{2}\right\|=1,\left\langle v_{1}, x-x_{1}^{\prime}\right\rangle=\left\|v_{1}\right\|\left\|x-x_{1}^{\prime}\right\|,\left\langle v_{2}, x-x_{2}^{\prime}\right\rangle=\left\|v_{2}\right\|\left\|x-x_{2}^{\prime}\right\| .
\end{gathered}
$$

Let $\operatorname{itr}_{w}[A, B](\bar{x})$ denote the exact upper bound of all $\left.\alpha \in\right] 0,1[$ such that the above sufficient condition for subtransversality is satisfied for some $\delta>0$, with the convention that the supremum over the empty set equals 0 .

To this end, the following question about the above result is of importance. Our subsequent analysis will give the negative answer to it (see Remark 6).

Question 1 Is the sufficient condition formulated in Proposition 2, i.e. $\operatorname{itr}_{w}[A, B](\bar{x})>0$, also necessary for subtransversality?

We next recall the central concept of this paper, i.e., the intrinsic transversality property of pairs of sets. Compared to its better known siblings recalled in Definition 1, intrinsic transversality came to life much later.

Definition 2 (intrinsic transversality in Euclidean spaces) [13, Definition 3.1] A pair of closed sets $\{A, B\}$ in a Euclidean space is intrinsically transversal at a point $\bar{x} \in A \cap B$ if there exists an angle $\alpha>0$ together with a number $\delta>0$ such that any two points $a \in$ $(A \backslash B) \cap \mathbb{B}_{\delta}(\bar{x})$ and $b \in(B \backslash A) \cap \mathbb{B}_{\delta}(\bar{x})$ cannot have difference $a-b$ simultaneously making an angle strictly less than $\alpha$ with the two proximal normal cones $N_{B}^{p}(b)$ and $-N_{A}^{p}(a)$.

The above property was originally introduced in 2015 by Drusvyatskiy et al. [13] as a sufficient condition for local linear convergence of the alternating projection algorithm for solving nonconvex feasibility problems in Euclidean spaces. As demonstrated by Ioffe [23], Kruger et al. [32, 35] and will also be confirmed in this paper, intrinsic transversality turns out to be an important qualification property in variational analysis. Kruger [32] recently extended and investigated intrinsic transversality in more general underlying spaces. ${ }^{5}$

Definition 3 (intrinsic transversality) [35, Definition 4(ii)] \& [32, Definition 2(ii)] ${ }^{6} \mathrm{~A}$ pair of closed sets $\{A, B\}$ is intrinsically transversal at a point $\bar{x} \in A \cap B$ if there exist numbers $\alpha \in] 0,1\left[\right.$ and $\delta>0$ such that $\left\|v_{1}+v_{2}\right\|>\alpha$ for all $a \in(A \backslash B) \cap \mathbb{B}_{\delta}(\bar{x})$, $b \in(B \backslash A) \cap \mathbb{B}_{\delta}(\bar{x}), x \in \mathbb{B}_{\delta}(\bar{x})$ with $x \neq a, x \neq b, 1-\delta<\frac{\|x-a\|}{\|x-b\|}<1+\delta$, and $v_{1} \in N_{A}(a) \backslash\{0\}, v_{2} \in N_{B}(b) \backslash\{0\}$ satisfying

$$
\left\|v_{1}\right\|+\left\|v_{2}\right\|=1, \frac{\left\langle v_{1}, x-a\right\rangle}{\left\|v_{1}\right\|\|x-a\|}>1-\delta, \frac{\left\langle v_{2}, x-b\right\rangle}{\left\|v_{2}\right\|\|x-b\|}>1-\delta .
$$

The exact upper bound of all $\alpha \in] 0,1[$ that together with some $\delta>0$ satisfies the above definition of intrinsic transversality is denoted by $\operatorname{itr}[A, B](\bar{x})$, with the convention that the supremum over the empty set equals 0 .

\footnotetext{
${ }^{5}$ It is worth noting that the extension from Definition 2 to Definition 3 of intrinsic transversality is not trivial, and the coincidence of the two definitions in the Euclidean space setting was shown in [32, Proposition 8(iii)]. ${ }^{6}$ The property was defined and investigated in general normed linear spaces.
} 
Remark 2 In Definition 3 it can be assumed without loss of generality that $\delta \in] 0,1[$. In this case, the three conditions $a \in A \backslash B, b \in B \backslash A$ and $1-\delta<\frac{\|x-a\|}{\|x-b\|}<1+\delta$ imply conditions $x \neq a$ and $x \neq b$. In similar contexts in the sequel, the latter two conditions will be omitted for the sake of brevity, for example, in the proofs of Lemmas $1 \& 2$, representation Eq. 72 and Definition 5(i).

Making use of the quantities $\operatorname{str}[A, B](\bar{x}), \operatorname{tr}[A, B](\bar{x}), \operatorname{itr}_{c}[A, B](\bar{x}), \operatorname{itr}_{w}[A, B](\bar{x})$ and $\operatorname{itr}[A, B](\bar{x})$, we can concisely summarize the facts recalled so far in this section. The definitions of subtransversality, transversality and intrinsic transversality and Propositions $1 \&$ 2 respectively admit more concise descriptions.

Proposition 3 (summary) Let $\{A, B\}$ be a pair of closed sets and $\bar{x} \in A \cap B$.

(i) $\{A, B\}$ is subtransversal at $\bar{x}$ if and only if $\operatorname{str}[A, B](\bar{x})>0$.

(ii) $\{A, B\}$ is transversal at $\bar{x}$ if and only if $\operatorname{tr}[A, B](\bar{x})>0$.

(iii) $\{A, B\}$ is intrinsically transversal at $\bar{x}$ if and only if $\operatorname{itr}[A, B](\bar{x})>0$.

(iv) If the sets are convex, then $\{A, B\}$ is subtransversal at $\bar{x}$ if and only if $\operatorname{itr}_{c}[A, B](\bar{x})>0$.

(v) $\{A, B\}$ is subtransversal at $\bar{x}$ if $\operatorname{itr}_{w}[A, B](\bar{x})>0$.

In this section, we are particularly interested in the result established by Kruger [32, Theorem 4] that intrinsic transversality implies the sufficient condition for subtransversality stated in Proposition 2, which in turn implies the one stated in Proposition 1. These implications are captured by Proposition 4(i) via the relationships between the corresponding quantities. For completeness, more comprehensive relationships between the transversality-type properties are also presented here.

Proposition 4 (relationships between quantitative constants) [32, Proposition 1] Let $\{A, B\}$ be a pair of closed sets and $\bar{x} \in A \cap B$.

(i) $0 \leq \operatorname{tr}[A, B](\bar{x}) \leq \operatorname{itr}[A, B](\bar{x}) \leq \operatorname{itr}_{w}[A, B](\bar{x}) \leq \operatorname{itr}_{c}[A, B](\bar{x}) \leq 1 .^{7}$

(ii) $\operatorname{itr}_{w}[A, B](\bar{x}) \leq \operatorname{str}[A, B](\bar{x}){ }^{8}$

(iii) If $A$ and $B$ are convex, then $\operatorname{itr}_{c}[A, B](\bar{x})=\operatorname{str}[A, B](\bar{x})$.

(iv) If $\operatorname{dim} X<\infty$ and $A, B$ are convex, then $\operatorname{itr}_{w}[A, B](\bar{x})=\operatorname{itr}_{c}[A, B](\bar{x})=$ $\operatorname{str}[A, B](\bar{x})$.

Remark 3 (notation and terminology) In view of Proposition 4(i)\&(ii), the strict inequality $\operatorname{itr}_{w}[A, B](\bar{x})>0$ corresponds to a property, which is weaker than intrinsic transversality and stronger than subtransversality. That property is called weak intrinsic transversality in $[32,35]$. This in particular explains why the letter " $w$ " is used in the notation $\operatorname{itr}_{w}[A, B](\bar{x})$. Similarly, the strict inequality $\operatorname{itr}_{c}[A, B](\bar{x})>0$ corresponds to a weaker property than weak intrinsic transversality. Such a property has not been named yet, but it has played an important role in the analysis of transversality-type properties mainly in the convex setting [32]. This particularly explains why the letter " $c$ " is used in the notation $\operatorname{itr}_{c}[A, B](\bar{x})$.

Proposition 4(iv) in particular claims the equivalence between weak intrinsic transversality and intrinsic transversality in the convex and finite dimensional setting. The following

\footnotetext{
${ }^{7}$ The statement is valid in Banach spaces.

${ }^{8}$ The statement is valid in Asplund spaces.
} 
question about their relationship in more general settings is of interest. Subsequent analysis will give the answer to this question in the Hilbert space setting (see Remark 5).

Question 2 [32, question 3, page 140] What is the relationship between weak intrinsic transversality and intrinsic transversality in the general nonconvex setting?

The next result establishes the main quantitative estimate of this section. Though the statement and its proof are rather technical, its meaningful consequences will follow shortly.

Lemma 1 (quantitative estimate) Let $\{A, B\}$ be a pair of closed sets and $\bar{x} \in A \cap B$. It holds

$$
\min \left\{\operatorname{itr}_{c}[A, B](\bar{x}), 1 / \sqrt{2}\right\} \leq \operatorname{itr}[A, B](\bar{x}) .
$$

Proof To proceed with the proof, let us suppose that $\operatorname{itr}_{c}[A, B](\bar{x})>0$ since there is nothing to prove in the case $\operatorname{itr}_{c}[A, B](\bar{x})=0$. Let us fix an arbitrary number

$$
\beta \in] 0, \min \left\{\operatorname{itr}_{c}[A, B](\bar{x}), 1 / \sqrt{2}\right\}[
$$

and prove that $\operatorname{itr}[A, B](\bar{x}) \geq \beta$. By the definition of $\operatorname{itr}_{c}[A, B](\bar{x})$, there exist numbers

$$
\alpha \in] \beta, \min \left\{\operatorname{itr}_{c}[A, B](\bar{x}), 1 / \sqrt{2}\right\}[
$$

and $\delta>0$ such that, for all $a \in(A \backslash B) \cap \mathbb{B}_{\delta}(\bar{x}), b \in(B \backslash A) \cap \mathbb{B}_{\delta}(\bar{x})$ and $x \in \mathbb{B}_{\delta}(\bar{x})$ with $\|x-a\|=\|x-b\|$, one has

$$
\left\|v_{1}+v_{2}\right\|>\alpha
$$

for all $v_{1}, v_{2} \in X$ satisfying

$$
\begin{gathered}
\operatorname{dist}\left(v_{1}, N_{A}(a)\right)<\delta, \operatorname{dist}\left(v_{2}, N_{B}(b)\right)<\delta, \\
\left\|v_{1}\right\|+\left\|v_{2}\right\|=1, \quad\left\langle v_{1}, x-a\right\rangle=\left\|v_{1}\right\|\|x-a\|,\left\langle v_{2}, x-b\right\rangle=\left\|v_{2}\right\|\|x-b\| .
\end{gathered}
$$

Choose a number $\left.\delta^{\prime} \in\right] 0, \delta / 3[$ and satisfying

$$
\begin{gathered}
2\left(\sqrt{\delta^{\prime}}+\delta^{\prime}\right)<1 / 2-\beta^{2}, \\
\sqrt{2 \delta^{\prime}}+2 \sqrt{\frac{2 \delta^{\prime}-\delta^{\prime 2}}{4-6 \delta^{\prime}+3 \delta^{\prime 2}}}<\min \{\delta, \alpha-\beta\} .
\end{gathered}
$$

Such a number $\delta^{\prime}$ exists since $1 / 2-\beta^{2}>0, \min \{\delta, \alpha-\beta\}>0$ and

$$
\lim _{t \downarrow 0} 2(\sqrt{t}+t)=0, \lim _{t \downarrow 0}\left(\sqrt{2 t}+2 \sqrt{\frac{2 t-t^{2}}{4-6 t+3 t^{2}}}\right)=0 .
$$

We are going to prove $\operatorname{itr}[A, B](\bar{x}) \geq \beta$ with the technical constant $\delta^{\prime}>0$. To begin, let us take any $a \in(A \backslash B) \cap \mathbb{B}_{\delta^{\prime}}(\bar{x}), b \in(B \backslash A) \cap \mathbb{B}_{\delta^{\prime}}(\bar{x})$ and $x \in \mathbb{B}_{\delta^{\prime}}(\bar{x})$ with $x \neq a, x \neq b$,

$$
1-\delta^{\prime}<\frac{\|x-a\|}{\|x-b\|}<1+\delta^{\prime}
$$

and $v_{1} \in N_{A}(a) \backslash\{0\}, v_{2} \in N_{B}(b) \backslash\{0\}$ satisfying

$$
\left\|v_{1}\right\|+\left\|v_{2}\right\|=1, \frac{\left\langle v_{1}, x-a\right\rangle}{\left\|v_{1}\right\|\|x-a\|}>1-\delta^{\prime}, \frac{\left\langle v_{2}, x-b\right\rangle}{\left\|v_{2}\right\|\|x-b\|}>1-\delta^{\prime} .
$$

All we need is to show that

$$
\left\|v_{1}+v_{2}\right\|>\beta
$$


We first observe from Eq. 14 that

$$
\begin{aligned}
& \left\|\frac{v_{1}}{\left\|v_{1}\right\|}-\frac{x-a}{\|x-a\|}\right\|^{2}=2-2 \frac{\left\langle v_{1}, x-a\right\rangle}{\left\|v_{1}\right\|\|x-a\|}<2-2\left(1-\delta^{\prime}\right)=2 \delta^{\prime}, \\
& \left\|\frac{v_{2}}{\left\|v_{2}\right\|}-\frac{x-b}{\|x-b\|}\right\|^{2}=2-2 \frac{\left\langle v_{2}, x-b\right\rangle}{\left\|v_{2}\right\|\|x-b\|}<2-2\left(1-\delta^{\prime}\right)=2 \delta^{\prime} .
\end{aligned}
$$

We take care of two possibilities concerning the value of $\langle x-a, x-b\rangle$ as follows.

Case 1. $\langle x-a, x-b\rangle>0$. Then

$$
\left\|\frac{x-a}{\|x-a\|}-\frac{x-b}{\|x-b\|}\right\|^{2}=2-2 \frac{\langle x-a, x-b\rangle}{\|x-a\|\|x-b\|}<2 .
$$

Equivalently,

$$
\left\|\frac{x-a}{\|x-a\|}-\frac{x-b}{\|x-b\|}\right\|<\sqrt{2}
$$

By the triangle inequality and estimates Eq. 17, Eq. 16, we get that

$$
\begin{aligned}
\left\|\frac{v_{1}}{\left\|v_{1}\right\|}-\frac{v_{2}}{\left\|v_{2}\right\|}\right\| & \leq\left\|\frac{x-a}{\|x-a\|}-\frac{x-b}{\|x-b\|}\right\|+\left\|\frac{v_{1}}{\left\|v_{1}\right\|}-\frac{x-a}{\|x-a\|}\right\|+\left\|\frac{v_{2}}{\left\|v_{2}\right\|}-\frac{x-b}{\|x-b\|}\right\| \\
& <\sqrt{2}+2 \sqrt{2 \delta^{\prime}}=\sqrt{2}\left(1+2 \sqrt{\delta^{\prime}}\right) .
\end{aligned}
$$

This implies that

$$
\begin{aligned}
\left\|\frac{v_{1}}{\left\|v_{1}\right\|}-\frac{v_{2}}{\left\|v_{2}\right\|}\right\|^{2} & =2-2 \frac{\left\langle v_{1}, v_{2}\right\rangle}{\left\|v_{1}\right\|\left\|v_{2}\right\|}<2\left(1+2 \sqrt{\delta^{\prime}}\right)^{2} \\
& \Leftrightarrow\left\langle v_{1}, v_{2}\right\rangle>-4\left(\sqrt{\delta^{\prime}}+\delta^{\prime}\right)\left\|v_{1}\right\|\left\|v_{2}\right\| .
\end{aligned}
$$

Using $\left\|v_{1}\right\|+\left\|v_{2}\right\|=1$ which implies $\left\|v_{1}\right\|\left\|v_{2}\right\| \leq 1 / 4$ and Eq. 18, respectively, we obtain that

$$
\begin{aligned}
\left\|v_{1}+v_{2}\right\|^{2} & =\left\|v_{1}\right\|^{2}+\left\|v_{2}\right\|^{2}+2\left\langle v_{1}, v_{2}\right\rangle=1-2\left\|v_{1}\right\|\left\|v_{2}\right\|+2\left\langle v_{1}, v_{2}\right\rangle \\
& >1-2\left\|v_{1}\right\|\left\|v_{2}\right\|-8\left(\sqrt{\delta^{\prime}}+\delta^{\prime}\right)\left\|v_{1}\right\|\left\|v_{2}\right\| \geq \frac{1}{2}-2\left(\sqrt{\delta^{\prime}}+\delta^{\prime}\right) .
\end{aligned}
$$

This, combined with Eq. 11 yields that

$$
\left\|v_{1}+v_{2}\right\|>\sqrt{\frac{1}{2}-2\left(\sqrt{\delta^{\prime}}+\delta^{\prime}\right)}>\beta .
$$

Case 2.

$$
\langle x-a, x-b\rangle \leq 0
$$

Let us define $m=\frac{a+b}{2}$ and

$$
x^{\prime}=x-\frac{\langle b-a, x-m\rangle}{\|b-a\|^{2}}(b-a) .
$$

We first check that

$$
\left\|x^{\prime}-a\right\|=\left\|x^{\prime}-b\right\|
$$


Indeed,

$$
\begin{aligned}
\left\|x^{\prime}-a\right\|^{2}-\left\|x^{\prime}-b\right\|^{2} & =\|x-a\|^{2}-\|x-b\|^{2}-2 \frac{\langle b-a, x-m\rangle}{\|b-a\|^{2}}\langle x-a, b-a\rangle \\
& +2 \frac{\langle b-a, x-m\rangle}{\|b-a\|^{2}}\langle x-b, b-a\rangle \\
& =\|x-a\|^{2}-\|x-b\|^{2}-2 \frac{\langle b-a, x-m\rangle}{\|b-a\|^{2}}\langle b-a, b-a\rangle \\
& =\|x-a\|^{2}-\|x-b\|^{2}-2\langle b-a, x-m\rangle \\
& =\|x-a\|^{2}-\|x-b\|^{2}-\langle(x-a)-(x-b),(x-a)+(x-b)\rangle=0 .
\end{aligned}
$$

We next check that

$$
\left\langle x-x^{\prime}, x^{\prime}-m\right\rangle=0 .
$$

Indeed, by Eq. 20, it holds that

$$
\left\langle x-x^{\prime}, x^{\prime}-m\right\rangle=\frac{\langle b-a, x-m\rangle}{\|b-a\|^{2}}\left\langle b-a, x^{\prime}-m\right\rangle,
$$

from which Eq. 22 follows since

$$
\begin{aligned}
\left\langle b-a, x^{\prime}-m\right\rangle & =\left\langle b-a, x-m-\frac{\langle b-a, x-m\rangle}{\|b-a\|^{2}}(b-a)\right\rangle \\
& =\langle b-a, x-m\rangle-\frac{\langle b-a, x-m\rangle}{\|b-a\|^{2}}\langle b-a, b-a\rangle=0 .
\end{aligned}
$$

Let us define also

$$
v_{1}^{\prime}=\frac{\left\|v_{1}\right\|}{\left\|x^{\prime}-a\right\|}\left(x^{\prime}-a\right), v_{2}^{\prime}=\frac{\left\|v_{2}\right\|}{\left\|x^{\prime}-b\right\|}\left(x^{\prime}-b\right) .
$$

It is clear that

$$
\begin{aligned}
& \left\|v_{1}^{\prime}\right\|=\left\|v_{1}\right\|,\left\|v_{2}^{\prime}\right\|=\left\|v_{2}\right\|,\left\|v_{1}^{\prime}\right\|+\left\|v_{2}^{\prime}\right\|=1, \\
& \left\langle v_{1}^{\prime}, x^{\prime}-a\right\rangle=\left\|v_{1}\right\|\left\|x^{\prime}-a\right\|=\left\|v_{1}^{\prime}\right\|\left\|x^{\prime}-a\right\|, \\
& \left\langle v_{2}^{\prime}, x^{\prime}-b\right\rangle=\left\|v_{2}\right\|\left\|x^{\prime}-b\right\|=\left\|v_{2}^{\prime}\right\|\left\|x^{\prime}-b\right\| .
\end{aligned}
$$

We next check that

$$
\operatorname{dist}\left(v_{1}^{\prime}, N_{A}(a)\right)<\delta, \operatorname{dist}\left(v_{2}^{\prime}, N_{B}(b)\right)<\delta .
$$

Let us prove $\operatorname{dist}\left(v_{1}^{\prime}, N_{A}(a)\right)<\delta$. Indeed, since $v_{1} \in N_{A}(a)$, it holds by Eq. 23 that

$$
\begin{aligned}
\operatorname{dist}\left(v_{1}^{\prime}, N_{A}(a)\right) & \leq\left\|v_{1}^{\prime}-v_{1}\right\|=\left\|\frac{\left\|v_{1}\right\|}{\left\|x^{\prime}-a\right\|}\left(x^{\prime}-a\right)-v_{1}\right\| \\
& =\left\|v_{1}\right\|\left\|\frac{x^{\prime}-a}{\left\|x^{\prime}-a\right\|}-\frac{v_{1}}{\left\|v_{1}\right\|}\right\| \\
& \leq\left\|v_{1}\right\|\left(\left\|\frac{x-a}{\|x-a\|}-\frac{v_{1}}{\left\|v_{1}\right\|}\right\|+\left\|\frac{x^{\prime}-a}{\left\|x^{\prime}-a\right\|}-\frac{x-a}{\|x-a\|}\right\|\right) .
\end{aligned}
$$

An upper bound of $\left\|\frac{x-a}{\|x-a\|}-\frac{v_{1}}{\left\|v_{1}\right\|}\right\|$ has been given by Eq. 16:

$$
\left\|\frac{x-a}{\|x-a\|}-\frac{v_{1}}{\left\|v_{1}\right\|}\right\|<\sqrt{2 \delta^{\prime}} .
$$

We now establish an upper bound of $\left\|\frac{x^{\prime}-a}{\left\|x^{\prime}-a\right\|}-\frac{x-a}{\|x-a\|}\right\|$ via three steps as follows. 
Step 1. We show that

$$
\left\|x-x^{\prime}\right\|^{2} \leq \frac{2 \delta^{\prime}-\delta^{\prime 2}}{4\left(1-\delta^{\prime}\right)^{2}} \min \left\{\|x-a\|^{2},\|x-b\|^{2}\right\} .
$$

If $\|x-a\| \geq\|x-b\|$, then

$$
\begin{gathered}
\|x-a\|^{2}-\|x-b\|^{2} \geq 0 \\
\Leftrightarrow\|x-m\|^{2}+\|m-a\|^{2}+2\langle x-m, m-a\rangle-\|x-m\|^{2}-\|m-b\|^{2} \\
-2\langle x-m, m-b\rangle \geq 0 \\
\Leftrightarrow\langle b-a, x-m\rangle \geq 0 .
\end{gathered}
$$

Note from Eq. 19 that

$$
\langle b-a, x-b\rangle=\langle b-x, x-b\rangle+\langle x-a, x-b\rangle=-\|x-b\|^{2}+\langle x-a, x-b\rangle \leq 0 .
$$

Taking Eq. 20, Eq. 29 and Eq. 30 into account, we have that

$$
\begin{aligned}
\left\|x^{\prime}-b\right\|^{2}-\|x-b\|^{2} & =\left\|x^{\prime}-x\right\|^{2}+2\left\langle x^{\prime}-x, x-b\right\rangle \\
& =\left\|x^{\prime}-x\right\|^{2}-2 \frac{\langle b-a, x-m\rangle}{\|b-a\|^{2}}\langle b-a, x-b\rangle \\
& \geq\left\|x^{\prime}-x\right\|^{2} .
\end{aligned}
$$

By Eq. 21 and Eq. 22 we get that

$$
\begin{aligned}
\|x-a\|^{2}+\|x-b\|^{2} & =2\left\|x-x^{\prime}\right\|^{2}+\left\|x^{\prime}-a\right\|^{2}+\left\|x^{\prime}-b\right\|^{2}+2\left\langle x-x^{\prime}, 2 x^{\prime}-(a+b)\right\rangle \\
& =2\left\|x-x^{\prime}\right\|^{2}+2\left\|x^{\prime}-b\right\|^{2}+4\left\langle x-x^{\prime}, x^{\prime}-m\right\rangle \\
& =2\left\|x-x^{\prime}\right\|^{2}+2\left\|x^{\prime}-b\right\|^{2} .
\end{aligned}
$$

This together with Eq. 13 and Eq. 31 yields that

$$
\begin{aligned}
2\left\|x-x^{\prime}\right\|^{2} & =\|x-a\|^{2}+\|x-b\|^{2}-2\left\|x^{\prime}-b\right\|^{2} \\
& \leq\left(1+\delta^{\prime}\right)^{2}\|x-b\|^{2}+\|x-b\|^{2}-2\left\|x^{\prime}-b\right\|^{2} \\
& \leq\left(1+\delta^{\prime}\right)^{2}\|x-b\|^{2}+\|x-b\|^{2}-2\left(\|x-b\|^{2}+\left\|x^{\prime}-x\right\|^{2}\right) .
\end{aligned}
$$

Equivalently,

$$
4\left\|x-x^{\prime}\right\|^{2} \leq\left(2 \delta^{\prime}+\delta^{\prime 2}\right)\|x-b\|^{2}=\left(2 \delta^{\prime}+\delta^{\prime 2}\right) \min \left\{\|x-a\|^{2},\|x-b\|^{2}\right\}
$$

since $\|x-a\| \geq\|x-b\|$ in this case.

By a similar argument, if $\|x-a\| \leq\|x-b\|$, then

$$
\langle b-a, x-m\rangle \leq 0,\langle b-a, x-a\rangle \geq 0 .
$$

Thus

$$
\begin{aligned}
\left\|x^{\prime}-a\right\|^{2}-\|x-a\|^{2} & =\left\|x^{\prime}-x\right\|^{2}+2\left\langle x^{\prime}-x, x-a\right\rangle \\
& =\left\|x^{\prime}-x\right\|^{2}-2 \frac{\langle b-a, x-m\rangle}{\|b-a\|^{2}}\langle b-a, x-a\rangle \\
& \geq\left\|x^{\prime}-x\right\|^{2} .
\end{aligned}
$$

By Eq. 21 and Eq. 22 we get that

$$
\|x-a\|^{2}+\|x-b\|^{2}=2\left\|x-x^{\prime}\right\|^{2}+2\left\|x^{\prime}-a\right\|^{2},
$$


which together with Eq. 13 and Eq. 33 yields that

$$
2\left\|x-x^{\prime}\right\|^{2} \leq\|x-a\|^{2}+\frac{1}{\left(1-\delta^{\prime}\right)^{2}}\|x-a\|^{2}-2\left(\|x-a\|^{2}+\left\|x^{\prime}-x\right\|^{2}\right) .
$$

Equivalently,

$$
4\left\|x-x^{\prime}\right\|^{2} \leq \frac{2 \delta^{\prime}-\delta^{\prime 2}}{\left(1-\delta^{\prime}\right)^{2}}\|x-a\|^{2}=\frac{2 \delta^{\prime}-\delta^{\prime 2}}{\left(1-\delta^{\prime}\right)^{2}} \min \left\{\|x-a\|^{2},\|x-b\|^{2}\right\}
$$

since $\|x-a\| \leq\|x-b\|$ in this case.

Combining Eq. 32 and Eq. 34 and noting that $2 \delta^{\prime}+\delta^{\prime 2}<\frac{2 \delta^{\prime}-\delta^{\prime 2}}{\left(1-\delta^{\prime}\right)^{2}}$, we obtain Eq. 28 as claimed.

Step 2. We show that

$$
\left\|x^{\prime}-a\right\|^{2} \geq \frac{4-6 \delta^{\prime}+3 \delta^{\prime 2}}{2 \delta^{\prime}-\delta^{\prime 2}}\left\|x-x^{\prime}\right\|^{2} .
$$

Indeed, if $\|x-a\| \leq\|x-b\|$, then the use of Eq. 33 and Eq. 34 yields Eq. 35:

$$
\begin{aligned}
\left\|x^{\prime}-a\right\|^{2} & \geq\|x-a\|^{2}+\left\|x-x^{\prime}\right\|^{2} \\
& \geq \frac{\left.4\left(1-\delta^{\prime}\right)^{2}\right)}{2 \delta^{\prime}-\delta^{\prime 2}}\left\|x-x^{\prime}\right\|^{2}+\left\|x-x^{\prime}\right\|^{2}=\frac{4-6 \delta^{\prime}+3 \delta^{\prime 2}}{2 \delta^{\prime}-\delta^{\prime 2}}\left\|x-x^{\prime}\right\|^{2} .
\end{aligned}
$$

Otherwise, i.e., $\|x-a\| \geq\|x-b\|$, then the use of Eq. 21, Eq. 31 and Eq. 32 successively implies that

$$
\begin{aligned}
\left\|x^{\prime}-a\right\|^{2} & =\left\|x^{\prime}-b\right\|^{2} \geq\|x-b\|^{2}+\left\|x-x^{\prime}\right\|^{2} \\
& \geq \frac{4}{2 \delta^{\prime}+\delta^{\prime 2}}\left\|x-x^{\prime}\right\|^{2}+\left\|x-x^{\prime}\right\|^{2}=\frac{4+2 \delta^{\prime}+\delta^{\prime 2}}{2 \delta^{\prime}+\delta^{\prime 2}}\left\|x-x^{\prime}\right\|^{2},
\end{aligned}
$$

which also yields Eq. 35 since $\frac{4+2 \delta^{\prime}+\delta^{\prime 2}}{2 \delta^{\prime}+\delta^{\prime 2}}>\frac{4-6 \delta^{\prime}+3 \delta^{\prime 2}}{2 \delta^{\prime}-\delta^{\prime 2}}$. Hence Eq. 35 has been proved.

Step 3. We show that

$$
\left\|\frac{x^{\prime}-a}{\left\|x^{\prime}-a\right\|}-\frac{x-a}{\|x-a\|}\right\| \leq 2 \frac{\left\|x-x^{\prime}\right\|}{\left\|x^{\prime}-a\right\|} .
$$

Indeed,

$$
\begin{aligned}
\left\|\frac{x^{\prime}-a}{\left\|x^{\prime}-a\right\|}-\frac{x-a}{\|x-a\|}\right\| & \leq\left\|\frac{x^{\prime}-a}{\left\|x^{\prime}-a\right\|}-\frac{x-a}{\left\|x^{\prime}-a\right\|}\right\|+\left\|\frac{x-a}{\left\|x^{\prime}-a\right\|}-\frac{x-a}{\|x-a\|}\right\| \\
& =\frac{\left\|x-x^{\prime}\right\|}{\left\|x^{\prime}-a\right\|}+\left|\frac{\|x-a\|}{\left\|x^{\prime}-a\right\|}-1\right| .
\end{aligned}
$$

If $\|x-a\| \geq\left\|x^{\prime}-a\right\|$, then Eq. 36 holds true since

$$
\left|\frac{\|x-a\|}{\left\|x^{\prime}-a\right\|}-1\right|=\frac{\|x-a\|}{\left\|x^{\prime}-a\right\|}-1 \leq \frac{\left\|x-x^{\prime}\right\|+\left\|x^{\prime}-a\right\|}{\left\|x^{\prime}-a\right\|}-1=\frac{\left\|x-x^{\prime}\right\|}{\left\|x^{\prime}-a\right\|} .
$$

Otherwise, i.e., $\|x-a\|<\left\|x^{\prime}-a\right\|$, then Eq. 36 also holds true since

$$
\left|\frac{\|x-a\|}{\left\|x^{\prime}-a\right\|}-1\right|=1-\frac{\|x-a\|}{\left\|x^{\prime}-a\right\|} \leq 1-\frac{\left\|x^{\prime}-a\right\|-\left\|x-x^{\prime}\right\|}{\left\|x^{\prime}-a\right\|}=\frac{\left\|x-x^{\prime}\right\|}{\left\|x^{\prime}-a\right\|} .
$$

Hence Eq. 36 has been proved. 
A combination of Eq. 35 and Eq. 36 yields that

$$
\left\|\frac{x^{\prime}-a}{\left\|x^{\prime}-a\right\|}-\frac{x-a}{\|x-a\|}\right\| \leq 2 \sqrt{\frac{2 \delta^{\prime}-\delta^{\prime 2}}{4-6 \delta^{\prime}+3 \delta^{\prime 2}}} .
$$

Plugging Eq. 27 and Eq. 37 into Eq. 26 and using Eq. 12, we obtain that

$$
\begin{aligned}
\operatorname{dist}\left(v_{1}^{\prime}, N_{A}(a)\right) \leq\left\|v_{1}^{\prime}-v_{1}\right\| & \leq\left\|v_{1}\right\|\left(\sqrt{2 \delta^{\prime}}+2 \sqrt{\frac{2 \delta^{\prime}-\delta^{\prime 2}}{4-6 \delta^{\prime}+3 \delta^{\prime 2}}}\right) \\
& <\sqrt{2 \delta^{\prime}}+2 \sqrt{\frac{2 \delta^{\prime}-\delta^{\prime 2}}{4-6 \delta^{\prime}+3 \delta^{\prime 2}}}<\delta .
\end{aligned}
$$

The proof of $\operatorname{dist}\left(v_{2}^{\prime}, N_{B}(b)\right)<\delta$ is analogous, and we also obtain that

$$
\begin{aligned}
\operatorname{dist}\left(v_{2}^{\prime}, N_{B}(b)\right) \leq\left\|v_{2}^{\prime}-v_{2}\right\| & \leq\left\|v_{2}\right\|\left(\sqrt{2 \delta^{\prime}}+2 \sqrt{\frac{2 \delta^{\prime}-\delta^{\prime 2}}{4-6 \delta^{\prime}+3 \delta^{\prime 2}}}\right) \\
& <\sqrt{2 \delta^{\prime}}+2 \sqrt{\frac{2 \delta^{\prime}-\delta^{\prime 2}}{4-6 \delta^{\prime}+3 \delta^{\prime 2}}}<\delta .
\end{aligned}
$$

Hence Eq. 25 has been proved.

Conditions Eq. 25 and Eq. 24 ensure that the pair of vectors $\left\{v_{1}^{\prime}, v_{2}^{\prime}\right\}$ satisfies conditions Eq. 9 and Eq. 10, respectively. It is trivial from the choice of $\delta^{\prime}$ in Eq. 12 that $a \in(A \backslash B) \cap$ $\mathbb{B}_{\delta}(\bar{x}), b \in(B \backslash A) \cap \mathbb{B}_{\delta}(\bar{x})$. We also have $x^{\prime} \in \mathbb{B}_{\delta}(\bar{x})$ since

$$
\begin{aligned}
\left\|x^{\prime}-\bar{x}\right\| & =\left\|x-\frac{\langle b-a, x-m\rangle}{\|b-a\|^{2}}(b-a)-\bar{x}\right\| \leq\|x-\bar{x}\|+\|x-m\| \\
& \leq \delta^{\prime}+\max \{\|x-a\|,\|x-b\|\} \\
& \leq \delta^{\prime}+\|x-\bar{x}\|+\max \{\|a-\bar{x}\|,\|b-\bar{x}\|\} \leq 3 \delta^{\prime}<\delta .
\end{aligned}
$$

Hence, the estimate Eq. 8 is applicable to $\left\{v_{1}^{\prime}, v_{2}^{\prime}\right\}$. That is,

$$
\left\|v_{1}^{\prime}+v_{2}^{\prime}\right\|>\alpha \text {. }
$$

Now using the triangle inequality, Eq. 38, Eq. 39, Eq. 40, Eq. 12 and Eq. 7 successively, we obtain the desired estimate:

$$
\begin{aligned}
\left\|v_{1}+v_{2}\right\| & =\left\|v_{1}^{\prime}+v_{2}^{\prime}+v_{1}-v_{1}^{\prime}+v_{2}-v_{2}^{\prime}\right\| \\
& \geq\left\|v_{1}^{\prime}+v_{2}^{\prime}\right\|-\left\|v_{1}^{\prime}-v_{1}\right\|-\left\|v_{2}^{\prime}-v_{2}\right\| \\
& \geq \alpha-\left(\left\|v_{1}\right\|+\left\|v_{2}\right\|\right)\left(\sqrt{2 \delta^{\prime}}+2 \sqrt{\frac{2 \delta^{\prime}+\delta^{\prime 2}}{4+2 \delta^{\prime}+\delta^{\prime 2}}}\right) \\
& =\alpha-\left(\sqrt{2 \delta^{\prime}}+2 \sqrt{\frac{2 \delta^{\prime}-\delta^{\prime 2}}{4-6 \delta^{\prime}+3 \delta^{\prime 2}}}\right) \\
& >\alpha-(\alpha-\beta)=\beta .
\end{aligned}
$$

This completes Case 2 and Eq. 15 has been proved.

Hence, we have proved that $\{A, B\}$ is intrinsically transversal at $\bar{x}$ with $\operatorname{itr}[A, B](\bar{x}) \geq \beta$. Since $\beta$ can be arbitrarily close to $\min \left\{\operatorname{itr}_{c}[A, B](\bar{x}), 1 / \sqrt{2}\right\}$, we also obtain the estimate Eq. 6 and the proof is complete. 
Remark 4 The idea behind Lemma 1 comes from two observations. First, when pairs of vectors $\left(v_{1}, v_{2}\right)$ appearing in the definitions of $\operatorname{itr}[A, B](\bar{x})$ and $\operatorname{itr}_{c}[A, B](\bar{x})$ are further restricted to the constraint $\left\langle v_{1}, v_{2}\right\rangle<0$, the two groups of conditions defining the two constants become equivalent. Second, this additional constraint, which also gives rise to the number $1 / \sqrt{2}$ in Lemma 1 , does not qualitatively affect the properties characterized by these constants. See also Lemma 3 for a geometric counterpart of this constraint in the Euclidean space setting.

Combining Lemma 1 with Proposition 4, we obtain the main result of this section.

Theorem 1 (complete quantitative relationships) Let $\{A, B\}$ be a pair of closed sets and $\bar{x} \in A \cap B$. Then the following statements hold true.

(i) If $\operatorname{itr}_{c}[A, B](\bar{x}) \geq 1 / \sqrt{2}$, then

$$
\min \left\{\operatorname{itr}[A, B](\bar{x}), \operatorname{itr}_{w}[A, B](\bar{x}), \operatorname{itr}_{c}[A, B](\bar{x})\right\} \geq 1 / \sqrt{2} .
$$

(ii) If $\operatorname{itr}_{c}[A, B](\bar{x})<1 / \sqrt{2}$, then

$$
\operatorname{itr}_{c}[A, B](\bar{x})=\operatorname{itr}[A, B](\bar{x})=\operatorname{itr}_{w}[A, B](\bar{x}) .
$$

As a consequence, the three transversality-type properties characterized by the above constants are equivalent. In particular, $\operatorname{it}_{c}[A, B](\bar{x})$ is a refined equivalent qualitative constant of intrinsic transversality.

Proof (i) In this case, it holds $\min \left\{\operatorname{itr}_{c}[A, B](\bar{x}), 1 / \sqrt{2}\right\}=1 / \sqrt{2}$. Lemma 1 then yields $\operatorname{itr}[A, B](\bar{x}) \geq 1 / \sqrt{2}$, which in turn implies Eq. 41 because the left-hand side of Eq. 41 equals $\operatorname{itr}[A, B](\bar{x})$ in view of Proposition 4(i).

(ii) In this case, it holds $\min \left\{\operatorname{itr}_{c}[A, B](\bar{x}), 1 / \sqrt{2}\right\}=\operatorname{itr}_{c}[A, B](\bar{x})$. Lemma 1 then implies $\operatorname{itr}_{c}[A, B](\bar{x}) \leq \operatorname{itr}[A, B](\bar{x})$, which together with Proposition 4(i) yields the equalities in Eq. 42.

In view of Theorem 1, the following result covers both Propositions $1 \& 2$ in the Hilbert space setting. More importantly, it refines Proposition 2 which establishes the weakest sufficient condition in terms of normal vectors for subtransversality in the nonconvex setting.

Corollary 1 (refined sufficient condition for subtransversality) A pair of closed sets $\{A, B\}$ is subtransversal at $\bar{x} \in A \cap B$ if $\operatorname{itr}_{c}[A, B](\bar{x})>0$. The converse implication is also true when the sets are convex.

Proof Let us suppose $\operatorname{itr}_{c}[A, B](\bar{x})>0$. In both cases of either $\operatorname{itr}_{c}[A, B](\bar{x}) \geq 1 / \sqrt{2}$ or $\operatorname{itr}_{c}[A, B](\bar{x})<1 / \sqrt{2}$, Theorem 1 ensures that $\operatorname{itr}_{w}[A, B](\bar{x})>0$. In view of Proposition 4(ii), the latter implies subtransversality of $\{A, B\}$ at $\bar{x}$. The statement about the converse implication in the convex setting follows from Proposition 1.

Corollary 1 gives rise to the following question, which will be addressed in Remark 7.

Question 3 Is the sufficient condition $\operatorname{itr}_{c}[A, B](\bar{x})>0$ also necessary for subtransversality in the nonconvex setting?

The following result is of importance. 
Corollary 2 (intrinsic transversality is subtransversality in the convex setting) For pairs of closed and convex sets, subtransversality and intrinsic transversality are equivalent.

Proof In view of Corollary 1 , a pair of closed and convex sets $\{A, B\}$ is subtransversal at $\bar{x} \in A \cap B$ if and only if $\operatorname{itr}_{c}[A, B](\bar{x})>0$. By Theorem 1, this is equivalent to $\operatorname{itr}[A, B](\bar{x})>0$, i.e., $\{A, B\}$ is intrinsically transversal at $\bar{x}$.

As by-products, we address Questions 1 - 3 in the remainder of this section. It is worth mentioning that Question 2 remains open in more general underlying spaces.

Remark 5 (answer to Question 2) Theorem 1 clearly shows that weak intrinsic transversality (i.e., $\operatorname{itr}_{w}[A, B](\bar{x})>0$ ) and intrinsic transversality (i.e., $\operatorname{itr}[A, B](\bar{x})>0$ ) are equivalent in the Hilbert space setting. Note that in the case $\operatorname{itr}_{c}[A, B](\bar{x})>1 / \sqrt{2}$, the equality of $\operatorname{itr}_{w}[A, B](\bar{x})$ and $\operatorname{itr}[A, B](\bar{x})$ remains unknown.

Remark 6 (answer to Question 1) In view of Remark 5, the condition $\operatorname{itr}_{w}[A, B](\bar{x})>0$ (i.e, weak intrinsic transversality) is equivalent to intrinsic transversality of $\{A, B\}$ at $\bar{x}$. But it is widely known that the latter is not necessary for subtransversality in the nonconvex setting $[14,36,48]$. This establishes the negative answer to Question 1.

Remark 7 (answer to Question 3) Thanks to Theorem 1, the two conditions $\operatorname{itr}_{c}[A, B](\bar{x})>$ 0 and $\operatorname{itr}_{w}[A, B](\bar{x})>0$ are equivalent. The latter is not necessary for subtransversality as explained in Remark 6. As a consequence, the condition $\operatorname{itr}_{c}[A, B](\bar{x})>0$ is not necessary for subtransversality in the nonconvex setting.

In conclusion, Theorem 1 unifies and refines a number of transversality-type properties in Hilbert spaces including intrinsic transversality [13], weak intrinsic transversality [32], the sufficient conditions for subtransversality [35, 36, 39] and the characterization of subtransversality with convexity [32]. This result significantly clarifies the picture of transversality-type properties.

\section{Intrinsic Transversality in Primal Space Terms}

In view of Corollary 2, for pairs of closed and convex sets in Hilbert spaces, intrinsic transversality is equivalent to subtransversality which is defined in primal space terms. ${ }^{9}$ The situation for pairs of nonconvex sets has not been known and requires clarification; see, for example, the following question raised by Ioffe [22, Remark 6.1].

\section{Question 4 [22] What are primal space counterparts of intrinsic transversality?}

The analysis in this section is devoted to addressing the above question. The main goal is to characterize intrinsic transversality by conditions which do not involve normal vectors.

\footnotetext{
${ }^{9}$ In this section, the term primal space is used to indicate that the mentioned objects, conditions, properties or representations do not explicitly involve normal vectors.
} 
For convenience, let us introduce the notation involving three sets as follows: ${ }^{10}$

$$
\mathrm{d}(A, B, \Omega):=\inf _{x \in \Omega, a \in A, b \in B} \max \{\|x-a\|,\|x-b\|\}, \quad \text { for } A, B, \Omega \subset X,
$$

with the convention that the infimum over the empty set equals infinity.

Definition 4 (property $(\mathcal{P})^{11}$ ) A pair of closed sets $\{A, B\}$ is said to satisfy property $(\mathcal{P})$ at a point $\bar{x} \in A \cap B$ if there are numbers $\alpha \in] 0,1[$ and $\varepsilon>0$ such that for any $a \in$ $(A \backslash B) \cap \mathbb{B}_{\varepsilon}(\bar{x}), b \in(B \backslash A) \cap \mathbb{B}_{\varepsilon}(\bar{x})$ and $x \in \mathbb{B}_{\varepsilon}(\bar{x})$ with $\|x-a\|=\|x-b\|$ and number $\delta>0$, there exists $\rho \in] 0, \delta[$ satisfying

$$
\mathrm{d}\left(A \cap \overline{\mathbb{B}}_{\lambda}(a), B \cap \overline{\mathbb{B}}_{\lambda}(b), \mathbb{B}_{\rho}(x)\right)+\alpha \rho \leq\|x-a\|, \text { where } \lambda:=(\alpha+1 / \sqrt{\varepsilon}) \rho .
$$

The exact upper bound of all $\alpha \in] 0,1[\operatorname{such}$ that $\{A, B\}$ satisfies property $(\mathcal{P})$ at $\bar{x}$ for $\alpha$ and some $\varepsilon>0$ is denoted by $\operatorname{itr}_{p}[A, B](\bar{x})$.

The following statement is straightforward from the definition.

Proposition 5 A pair of closed sets $\{A, B\}$ satisfies property $(\mathcal{P})$ at a point $\bar{x} \in A \cap B$ if and only if $\operatorname{itr}_{p}[A, B](\bar{x})>0$.

We next formulate several technical results which are essential for proving the equivalence between property $(\mathcal{P})$ and intrinsic transversality.

Proposition 6 [8, Corollary 6.3 $]^{12}$ Let $\{A, B\}$ be a pair of closed sets in $X, \bar{x} \in A \cap B$, $u, v \in X$ and numbers $\rho, \varepsilon>0$. Suppose that

$$
\begin{gathered}
(A-u) \cap(B-v) \cap \mathbb{B}_{\rho}(\bar{x})=\emptyset, \\
\max \{\|u\|,\|v\|\}<\mathrm{d}\left(A-u, B-v, \mathbb{B}_{\rho}(\bar{x})\right)+\varepsilon .
\end{gathered}
$$

Then for any numbers $\lambda \geq \varepsilon+\rho$ and $\tau \in\left[0, \frac{\lambda-\varepsilon}{\lambda+\varepsilon}\left[\right.\right.$, there exist points $\hat{a} \in A \cap \mathbb{B}_{\lambda}(\bar{x})$, $\hat{b} \in B \cap \mathbb{B}_{\lambda}(\bar{x}), \hat{x} \in \mathbb{B}_{\rho}(\bar{x})$ and vectors $v_{1} \in N_{A}(\hat{a}), v_{2} \in N_{B}(\hat{b})$ such that

$$
\begin{gathered}
\left\|v_{1}\right\|+\left\|v_{2}\right\|=1,\left\|v_{1}+v_{2}\right\|<\varepsilon / \rho, \\
\left\langle v_{1}, \hat{x}-\hat{a}+u\right\rangle+\left\langle v_{2}, \hat{x}-\hat{b}+v\right\rangle>\tau \max \{\|\hat{x}-\hat{a}+u\|,\|\hat{x}-\hat{b}+v\|\} .
\end{gathered}
$$

Remark 8 Condition Eq. 47 plays an important role in searching for primal space counterparts of intrinsic transversality because it relates normal vectors to the primal space elements.

Our subsequent analysis requires the following modified version of Proposition 6, where the reference point $\bar{x} \in A \cap B$ is replaced by a triple of points $(a, b, x) \in A \times B \times X$.

\footnotetext{
${ }^{10}$ This is the distance between the two sets $A \times B$ and $\{(x, x) \in X \times X \mid x \in \Omega\}$ in $X \times X$ endowed with the maximum norm.

${ }^{11}$ The definition is valid in normed linear spaces.

${ }^{12}$ The result is valid in Asplund spaces.
} 
Proposition 7 Let $\{A, B\}$ be a pair of closed sets in $X, a \in A, b \in B, x \in X$ and numbers $\rho, \varepsilon>0$. Suppose that

$$
\begin{gathered}
0<\mathrm{d}\left(A, B, \mathbb{B}_{\rho}(x)\right), \\
\max \{\|x-a\|,\|x-b\|\}<\mathrm{d}\left(A, B, \mathbb{B}_{\rho}(x)\right)+\varepsilon .
\end{gathered}
$$

Then for any numbers $\lambda \geq \varepsilon+\rho$ and $\tau \in\left[0, \frac{\lambda-\varepsilon}{\lambda+\varepsilon}\left[\right.\right.$, there exist points $\hat{a} \in A \cap \mathbb{B}_{\lambda}(a)$, $\hat{b} \in B \cap \mathbb{B}_{\lambda}(b), \hat{x} \in \mathbb{B}_{\rho}(x)$ and vectors $v_{1} \in N_{A}(\hat{a}), v_{2} \in N_{B}(\hat{b})$ satisfying Eq. 46 and

$$
\left\langle v_{1}, \hat{x}-\hat{a}\right\rangle+\left\langle v_{2}, \hat{x}-\hat{b}\right\rangle>\tau \max \{\|\hat{x}-\hat{a}\|,\|\hat{x}-\hat{b}\|\} .
$$

Proof The idea of the proof is to apply Proposition 6 to

$$
A^{\prime}:=A-a, B^{\prime}:=B-b, \bar{x}:=0 \in A^{\prime} \cap B^{\prime}, u:=x-a \text { and } v:=x-b
$$

by verifying Eq. 44 and Eq. 45 . Indeed, condition Eq. 48 implies that

$$
A \cap B \cap \mathbb{B}_{\rho}(x)=\emptyset \text {, or equivalently, }(A-x) \cap(B-x) \cap(\rho \mathbb{B})=\emptyset .
$$

The latter is exactly Eq. 44 since by Eq. 51

$$
\left(A^{\prime}-u\right) \cap\left(B^{\prime}-v\right) \cap \mathbb{B}_{\rho}(\bar{x})=(A-x) \cap(B-x) \cap(\rho \mathbb{B}) .
$$

Note also from Eq. 51 that

$$
\mathrm{d}\left(A^{\prime}-u, B^{\prime}-v, \mathbb{B}_{\rho}(\bar{x})\right)=\mathrm{d}(A-x, B-x, \rho \mathbb{B})=\mathrm{d}\left(A, B, \mathbb{B}_{\rho}(x)\right) .
$$

This together with Eq. 49 yields condition Eq. 45.

In view of Proposition 6, there exist points

$$
a^{\prime} \in A^{\prime} \cap(\lambda \mathbb{B}), b^{\prime} \in B^{\prime} \cap(\lambda \mathbb{B}), x^{\prime} \in \rho \mathbb{B}
$$

and vectors $v_{1} \in N_{A^{\prime}}\left(a^{\prime}\right), v_{2} \in N_{B^{\prime}}\left(b^{\prime}\right)$ satisfying conditions Eq. 46 and Eq. 47. Let us define

$$
\hat{a}:=a^{\prime}+a, \hat{b}:=b^{\prime}+b \text { and } \hat{x}:=x^{\prime}+x .
$$

This together with Eq. 51 and Eq. 52 ensures that $\hat{a} \in A \cap \mathbb{B}_{\lambda}(a), \hat{b} \in B \cap \mathbb{B}_{\lambda}(b)$ and $\hat{x} \in \mathbb{B}_{\rho}(x)$. Note also that

$$
\begin{gathered}
N_{A}(\hat{a})=N_{A^{\prime}}\left(a^{\prime}\right), N_{B}(\hat{b})=N_{B^{\prime}}\left(b^{\prime}\right), \\
\hat{x}-\hat{a}=x^{\prime}-a^{\prime}+x-a=x^{\prime}-a^{\prime}+u, \hat{x}-\hat{b}=x^{\prime}-b^{\prime}+x-b=x^{\prime}-b^{\prime}+v .
\end{gathered}
$$

The combination of Eq. 47 and Eq. 54 yields Eq. 50 while condition Eq. 53 ensures that $v_{1} \in N_{A}(\hat{a}), v_{2} \in N_{B}(\hat{b})$.

Therefore, the points $\hat{a}, \hat{b}, \hat{x}$ and vectors $v_{1}, v_{2}$ satisfy all the required conditions of the proposition. The proof is complete.

Another technical result is needed for our analysis. 
Proposition $8{ }^{13}$ Let $\{A, B\}$ be a pair of closed sets in $X, a \in A, b \in B, x \in X$ with $\|x-a\|=\|x-b\|>0, \alpha, \varepsilon>0$ and vectors $v_{1}, v_{2} \in X$ with $\left\|v_{1}\right\|+\left\|v_{2}\right\|=1$. Suppose that the following conditions are satisfied:

$$
\begin{gathered}
\left\|v_{1}+v_{2}\right\|+2 \varepsilon(\alpha+1 / \sqrt{\varepsilon})<\alpha, \\
\operatorname{dist}\left(v_{1}, N_{A}(a)\right)<\varepsilon, \operatorname{dist}\left(v_{2}, N_{B}(b)\right)<\varepsilon, \\
\left\langle v_{1}, x-a\right\rangle=\left\|v_{1}\right\|\|x-a\|,\left\langle v_{2}, x-b\right\rangle=\left\|v_{2}\right\|\|x-b\| .
\end{gathered}
$$

Then there exists a number $\delta>0$ such that

$$
\mathrm{d}\left(A \cap \overline{\mathbb{B}}_{\lambda}(a), B \cap \overline{\mathbb{B}}_{\lambda}(b), \mathbb{B}_{\rho}(x)\right)+\alpha \rho>\|x-a\| \text {, where } \lambda:=(\alpha+1 / \sqrt{\varepsilon}) \rho,
$$

holds true for all $\rho \in] 0, \delta[$.

Proof By Eq. 56, there exist vectors $u_{1} \in N_{A}(a)$ and $u_{2} \in N_{B}(b)$ such that

$$
\left\|v_{1}-u_{1}\right\|<\varepsilon,\left\|v_{2}-u_{2}\right\|<\varepsilon .
$$

Since $\alpha-\left\|v_{1}+v_{2}\right\|>2 \varepsilon(\alpha+1 / \sqrt{\varepsilon})$ due to Eq. 55, there are positive numbers $\alpha_{1}$ and $\alpha_{2}$ such that

$$
\alpha>\alpha_{1}>\alpha_{2}>\left\|v_{1}+v_{2}\right\|, \alpha_{1}-\alpha_{2}>2 \varepsilon(\alpha+1 / \sqrt{\varepsilon}) .
$$

Choose a number $\beta>0$ such that

$$
\beta<\frac{\alpha_{1}-\alpha_{2}}{\alpha+1 / \sqrt{\varepsilon}}-2 \varepsilon, \text { equivalently, } \alpha_{1}-\alpha_{2}-(2 \varepsilon+\beta)(\alpha+1 / \sqrt{\varepsilon})>0 .
$$

By the definition Eq. 2 of the Fréchet normal cone, there is a number $\delta^{\prime}>0$ such that

$$
\begin{aligned}
& \left\langle u_{1}, a^{\prime}-a\right\rangle \leq \frac{\beta}{2}\left\|a^{\prime}-a\right\|,\left\langle u_{2}, b^{\prime}-b\right\rangle \leq \frac{\beta}{2}\left\|b^{\prime}-b\right\|, \\
& \forall a^{\prime} \in A \cap \mathbb{B}_{\delta^{\prime}}(a), b^{\prime} \in B \cap \mathbb{B}_{\delta^{\prime}}(b) .
\end{aligned}
$$

Let us define

$$
\delta:=\frac{\delta^{\prime}}{\alpha+1 / \sqrt{\varepsilon}}>0
$$

and show that $\delta$ fulfills the requirement of the proposition. Indeed, let us suppose to the contrary that condition Eq. 58 is not satisfied for some $\rho \in] 0, \delta[$. That is,

$$
\mathrm{d}\left(A \cap \overline{\mathbb{B}}_{\lambda}(a), B \cap \overline{\mathbb{B}}_{\lambda}(b), \mathbb{B}_{\rho}(x)\right)+\rho \alpha \leq\|x-a\|, \text { where } \lambda:=(\alpha+1 / \sqrt{\varepsilon}) \rho .
$$

Since $\alpha_{1}<\alpha$, the above inequality ensures the existence of $\hat{a} \in A \cap \overline{\mathbb{B}}_{\lambda}(a), \hat{b} \in B \cap \overline{\mathbb{B}}_{\lambda}(b)$ and $\hat{x} \in \mathbb{B}_{\rho}(x)$ such that

$$
\max \{\|\hat{x}-\hat{a}\|,\|\hat{x}-\hat{b}\|\}<\|x-a\|-\rho \alpha_{1} .
$$

Note that $\lambda=(\alpha+1 / \sqrt{\varepsilon}) \rho<(\alpha+1 / \sqrt{\varepsilon}) \delta=\delta^{\prime}$ thanks to Eq. 63. Then in view of Eq. 62, we have

This implies that

$$
\left\langle u_{1}, \hat{a}-a\right\rangle \leq \frac{\beta}{2}\|\hat{a}-a\|,\left\langle u_{2}, \hat{b}-b\right\rangle \leq \frac{\beta}{2}\|\hat{b}-b\| .
$$

$$
\left\langle u_{1}, \hat{a}-a\right\rangle+\left\langle u_{2}, \hat{b}-b\right\rangle \leq \frac{\beta}{2}\|\hat{a}-a\|+\frac{\beta}{2}\|\hat{b}-b\| \leq \frac{\beta}{2} \lambda+\frac{\beta}{2} \lambda=\lambda \beta .
$$

By Eq. 57, $\left\|v_{1}\right\|+\left\|v_{2}\right\|=1$ and $\|x-a\|=\|x-b\|$, it holds

$$
\left\langle v_{1}, x-a\right\rangle+\left\langle v_{2}, x-b\right\rangle=\left\|v_{1}\right\|\|x-a\|+\left\|v_{2}\right\|\|x-b\|=\|x-a\| .
$$

\footnotetext{
${ }^{13}$ The result is valid in normed linear spaces.
} 
By Eq. 64 and $\left\|v_{1}\right\|+\left\|v_{2}\right\|=1$, it holds

$$
\left\langle v_{1}, \hat{x}-\hat{a}\right\rangle+\left\langle v_{2}, \hat{x}-\hat{b}\right\rangle \leq \max \{\|\hat{x}-\hat{a}\|,\|\hat{x}-\hat{b}\|\}<\|x-a\|-\rho \alpha_{1} .
$$

By the Cauchy-Schwarz inequality and $\alpha_{2}>\left\|v_{1}+v_{2}\right\|$ in view of Eq. 60, it holds

$$
\left\langle v_{1}+v_{2}, x-\hat{x}\right\rangle \leq\left\|v_{1}+v_{2}\right\|\|x-\hat{x}\|<\rho \alpha_{2} .
$$

By the Cauchy-Schwarz inequality and Eq. 59, it holds

$$
\left\langle v_{1}-u_{1}, \hat{a}-a\right\rangle+\left\langle v_{2}-u_{2}, \hat{b}-b\right\rangle \leq \varepsilon\|\hat{a}-a\|+\varepsilon\|\hat{b}-b\| \leq 2 \lambda \varepsilon .
$$

Adding Eq. 69 and Eq. 65 yields that

$$
\left\langle v_{1}, \hat{a}-a\right\rangle+\left\langle v_{2}, \hat{b}-b\right\rangle \leq 2 \lambda \varepsilon+\lambda \beta .
$$

Making use of Eq. 66, Eq. 67, Eq. 68, Eq. 70, $\lambda=(\alpha+1 / \sqrt{\varepsilon}) \rho$ and Eq. 61 successively, we come up with

$$
\begin{aligned}
\|x-a\| & =\left\langle v_{1}, x-a\right\rangle+\left\langle v_{2}, x-b\right\rangle \\
& =\left\langle v_{1}, \hat{x}-\hat{a}\right\rangle+\left\langle v_{2}, \hat{x}-\hat{b}\right\rangle+\left\langle v_{1}+v_{2}, x-\hat{x}\right\rangle+\left\langle v_{1}, \hat{a}-a\right\rangle+\left\langle v_{2}, \hat{b}-b\right\rangle \\
& <\|x-a\|-\rho \alpha_{1}+\rho \alpha_{2}+2 \lambda \varepsilon+\lambda \beta \\
& =\|x-a\|-\rho\left(\alpha_{1}-\alpha_{2}-(2 \varepsilon+\beta)(\alpha+1 / \sqrt{\varepsilon})\right)<\|x-a\|,
\end{aligned}
$$

which is a contradiction and hence the proof is complete.

Remark 9 Condition Eq. 58 holding true for all $\rho \in] 0, \delta[$ is the negation of condition Eq. 43 holding true for some $\rho \in] 0, \delta[$.

The next lemma establishes the key quantitative estimates of this section.

Lemma 2 (quantitative estimates ${ }^{14}$ ) Let $\{A, B\}$ be a pair of closed sets and $\bar{x} \in A \cap B$. Then

$$
\operatorname{itr}[A, B](\bar{x}) \leq \operatorname{itr}_{p}[A, B](\bar{x}) \leq \operatorname{itr}_{c}[A, B](\bar{x}) .
$$

Proof We first prove $\operatorname{itr}[A, B](\bar{x}) \leq \operatorname{itr}_{p}[A, B](\bar{x})$. Since the inequality becomes trivial when $\operatorname{itr}_{p}[A, B](\bar{x})=1$, we only need to prove the inequality for the case $\operatorname{itr}_{p}[A, B](\bar{x})<$ 1. We take an arbitrary number $\alpha$ satisfying $\operatorname{itr}_{p}[A, B](\bar{x})<\alpha<1$ and show that $\operatorname{itr}[A, B](\bar{x}) \leq \alpha$. To do this, let us first recall the following representation of $\operatorname{itr}[A, B](\bar{x})$ [35, Equation (72)]:

$$
\operatorname{itr}[A, B](\bar{x})=\liminf _{\substack{a \rightarrow \bar{x}, b \rightarrow \bar{x}, x \rightarrow \bar{x}, a \in A \backslash B, b \in B \backslash A \\ v_{1} \in N_{A}(a) \backslash\{0\}, v_{2} \in N_{B}(b) \backslash\{0\},\left\|v_{1}\right\|+\left\|v_{2}\right\|=1 \\\|x-a\|}}\left\|v_{1}+v_{2}\right\|,
$$

with the convention that the infimum over the empty set equals 1 . In view of Eq. 72, all we need is to show that for any (arbitrarily small) number $\varepsilon>0$, there exists a pair of vectors $\left(v_{1}, v_{2}\right)$ satisfying the constraints under the lim inf in Eq. 72 and $\left\|v_{1}+v_{2}\right\|<\alpha$.

By the definition of $\operatorname{itr}_{p}[A, B](\bar{x})$ and the inequality $\operatorname{itr}_{p}[A, B](\bar{x})<\alpha$, we have that for any number $\varepsilon>0$, there exist points $a \in(A \backslash B) \cap \mathbb{B}_{\varepsilon}(\bar{x}), b \in(B \backslash A) \cap \mathbb{B}_{\varepsilon}(\bar{x})$ and

\footnotetext{
${ }^{14}$ The first inequality in Eq. 71 holds true in Asplund spaces while the second one holds true in normed linear spaces.
} 
$x \in \mathbb{B}_{\varepsilon}(\bar{x})$ with $\|x-a\|=\|x-b\|$ and a number $\delta>0$ such that the inequality Eq. 58 holds true for all $\rho \in] 0, \delta[$.

Since $A \cap B$ is a closed set and $a, b \notin A \cap B$, there is a number $\gamma \in] 0, \varepsilon$ [ such that

$$
\mathbb{B}_{\gamma}(a) \cap(A \cap B)=\emptyset, \mathbb{B}_{\gamma}(b) \cap(A \cap B)=\emptyset .
$$

Let us take an arbitrary number $\varepsilon \in] 0,1[$ and choose a number $\rho>0$ satisfying

$$
\rho<\min \left\{\delta, \frac{\gamma}{\alpha+1 / \sqrt{\varepsilon}}, \varepsilon, \frac{\varepsilon\|x-a\|}{1+\alpha+1 / \sqrt{\varepsilon}}\right\} .
$$

Define a number $\varepsilon^{\prime}:=\alpha \rho>0$ and note from Eq. 74 that

$$
\varepsilon^{\prime}=\alpha \rho<(\alpha+1+1 / \sqrt{\varepsilon}) \rho<\varepsilon\|x-a\|<\|x-a\|=\max \{\|x-a\|,\|x-b\|\} .
$$

We are going to apply Proposition 7 to the sets $A^{\prime}:=A \cap \overline{\mathbb{B}}_{\lambda}(a), B^{\prime}:=B \cap \overline{\mathbb{B}}_{\lambda}(b)$, the points $a \in A^{\prime}, b \in B^{\prime}, x \in X$ and numbers $\rho, \varepsilon^{\prime}, \lambda$ and $\tau:=\frac{\lambda-2 \alpha \rho}{\lambda+\alpha \rho}$. Let us verify all the conditions of the proposition. First, the inequality Eq. 58 reduces to Eq. 49. It also implies Eq. 48 since $\mathrm{d}\left(A^{\prime}, B^{\prime}, \mathbb{B}_{\rho}(x)\right)>\|x-a\|-\alpha \rho>0$ thanks to Eq. 75. It is clear that $\varepsilon^{\prime}+\rho=(\alpha+1) \rho<(\alpha+1 / \sqrt{\varepsilon}) \rho=\lambda$ as $\left.\varepsilon \in\right] 0,1\left[\right.$ and $\tau=\frac{\lambda-2 \alpha \rho}{\lambda+\alpha \rho}<\frac{\lambda-\alpha \rho}{\lambda+\alpha \rho}=\frac{\lambda-\varepsilon^{\prime}}{\lambda+\varepsilon^{\prime}}$. We have checked all the conditions of Proposition 7. Therefore, in view of Proposition 7, there exist points $\hat{a} \in A^{\prime} \cap \mathbb{B}_{\lambda}(a), \hat{b} \in B^{\prime} \cap \mathbb{B}_{\lambda}(b), \hat{x} \in \mathbb{B}_{\rho}(x)$ and vectors $v_{1} \in N_{A^{\prime}}(\hat{a})$ and $v_{2} \in N_{B^{\prime}}(\hat{b})$ satisfying Eq. 50 and

$$
\left\|v_{1}\right\|+\left\|v_{2}\right\|=1,\left\|v_{1}+v_{2}\right\|<\frac{\varepsilon^{\prime}}{\rho}=\alpha .
$$

The following observations verify that the vectors $\left(v_{1}, v_{2}\right)$ in conjunction with the points $(\hat{a}, \hat{b}, \hat{x})$ fulfill the constraints under the liminf in Eq. 72 .

- By the triangle inequality and $\rho<\varepsilon$ due to Eq. 74, it holds that

$$
\begin{aligned}
& \|\bar{x}-\hat{x}\| \leq\|\bar{x}-x\|+\|x-\hat{x}\|<\varepsilon+\rho \leq 2 \varepsilon, \\
& \|\bar{x}-\hat{a}\| \leq\|\bar{x}-a\|+\|a-\hat{a}\|<\varepsilon+\lambda=\varepsilon+(\alpha+1 / \sqrt{\varepsilon}) \rho \leq(1+\alpha) \varepsilon+\sqrt{\varepsilon}, \\
& \|\bar{x}-\hat{b}\| \leq\|\bar{x}-b\|+\|b-\hat{b}\|<\varepsilon+\lambda=\varepsilon+(\alpha+1 / \sqrt{\varepsilon}) \rho \leq(1+\alpha) \varepsilon+\sqrt{\varepsilon} .
\end{aligned}
$$

This implies that $\hat{x} \rightarrow \bar{x}, \hat{a} \rightarrow \bar{x}, \hat{b} \rightarrow \bar{x}$ as $\varepsilon \downarrow 0$.

- $\quad$ Since $\lambda<\gamma$ by the choice of $\rho$ in Eq. 74, we have $\hat{a} \in A \cap \mathbb{B}_{\gamma}(a)$ which together with Eq. 73 implies that $\hat{a} \notin B$. That is $\hat{a} \in A \backslash B$. Similarly, we also have $\hat{b} \in B \backslash A$.

- It holds $v_{1} \neq 0$ and $v_{2} \neq 0$. Indeed, if otherwise, Eq. 76 implies $\left\|v_{1}+v_{2}\right\|=1<\alpha$, which is a contradiction to the inequality $\alpha \leq 1$.

- $\quad$ Since $\hat{a} \in A^{\prime} \cap \mathbb{B}_{\lambda}(a)=A \cap \mathbb{B}_{\lambda}(a)$ and $\hat{b} \in B^{\prime} \cap \mathbb{B}_{\lambda}(b)=B \cap \mathbb{B}_{\lambda}(b)$ by the definitions of $A^{\prime}$ and $B^{\prime}$, we have

$$
v_{1} \in N_{A^{\prime}}(\hat{a})=N_{A \cap \overline{\mathbb{B}}_{\lambda}(a)}(\hat{a})=N_{A}(\hat{a}), v_{2} \in N_{A^{\prime}}(\hat{a})=N_{B \cap \overline{\mathbb{B}}_{\lambda}(b)}(\hat{b})=N_{B}(\hat{b}) .
$$

- By the triangle inequality and $(\alpha+1+1 / \sqrt{\varepsilon}) \rho<\varepsilon\|x-a\|$ in view of Eq. 74, it holds that

$$
\begin{aligned}
\|\hat{x}-\hat{a}\| & \leq\|x-a\|+\|\hat{x}-x\|+\|a-\hat{a}\| \leq\|x-a\|+\varepsilon^{\prime}+\lambda \\
& =\|x-a\|+(\alpha+1+1 / \sqrt{\varepsilon}) \rho<(1+\varepsilon)\|x-a\|, \\
\|\hat{x}-\hat{a}\| & \geq\|x-a\|-\|\hat{x}-x\|-\|a-\hat{a}\| \geq\|x-a\|-\varepsilon^{\prime}-\lambda \\
& =\|x-a\|-(\alpha+1+1 / \sqrt{\varepsilon}) \rho>(1-\varepsilon)\|x-a\| .
\end{aligned}
$$

Hence, we have

$$
(1-\varepsilon)\|x-a\| \leq\|\hat{x}-\hat{a}\| \leq(1+\varepsilon)\|x-a\| .
$$


Using similar estimates, we also have

$$
(1-\varepsilon)\|x-b\| \leq\|\hat{x}-\hat{b}\| \leq(1+\varepsilon)\|x-b\| .
$$

The above estimates together with $\|x-a\|=\|x-b\| \neq 0$ imply

$$
\frac{1-\varepsilon}{1+\varepsilon} \leq \frac{\|\hat{x}-\hat{a}\|}{\|\hat{x}-\hat{b}\|} \leq \frac{1+\varepsilon}{1-\varepsilon},
$$

which in turn implies that $\frac{\|\hat{x}-\hat{a}\|}{\|\hat{x}-\hat{b}\|} \rightarrow 1$ as $\varepsilon \downarrow 0$.

- By Eq. 76, the Cauchy-Schwarz inequality, Eq. 50 and the definition of $\tau$, we have

$$
\begin{aligned}
1=\left\|v_{1}\right\|+\left\|v_{2}\right\| & \geq \frac{\left\langle v_{1}, \hat{x}-\hat{a}\right\rangle}{\|\hat{x}-\hat{a}\|}+\frac{\left\langle v_{2}, \hat{x}-\hat{b}\right\rangle}{\|\hat{x}-\hat{b}\|} \\
& \geq \frac{\left\langle v_{1}, \hat{x}-\hat{a}\right\rangle}{\max \{\|\hat{x}-\hat{a}\|,\|\hat{x}-\hat{b}\|\}}+\frac{\left\langle v_{2}, \hat{x}-\hat{b}\right\rangle}{\max \{\|\hat{x}-\hat{a}\|,\|\hat{x}-\hat{b}\|\}} \\
& >\tau=\frac{\lambda-2 \alpha \rho}{\lambda+\alpha \rho}=\frac{(\alpha+1 / \sqrt{\varepsilon}) \rho-2 \alpha \rho}{(\alpha+1 / \sqrt{\varepsilon}) \rho+\alpha \rho}=\frac{1 / \sqrt{\varepsilon}-\alpha}{1 / \sqrt{\varepsilon}+2 \alpha},
\end{aligned}
$$

which tends to 1 as $\varepsilon \downarrow 0$. Thus,

$$
\frac{\left\langle v_{1}, \hat{x}-\hat{a}\right\rangle}{\|\hat{x}-\hat{a}\|}+\frac{\left\langle v_{2}, \hat{x}-\hat{b}\right\rangle}{\|\hat{x}-\hat{b}\|} \rightarrow 1 \quad \text { as } \quad \varepsilon \downarrow 0 .
$$

Due to the Cauchy-Schwarz inequality and $\left\|v_{1}\right\|+\left\|v_{2}\right\|=1$, the above convergence happens if and only if

$$
\frac{\left\langle v_{1}, \hat{x}-\hat{a}\right\rangle}{\left\|v_{1}\right\|\|\hat{x}-\hat{a}\|} \rightarrow 1 \quad \text { and } \quad \frac{\left\langle v_{2}, \hat{x}-\hat{b}\right\rangle}{\left\|v_{2}\right\|\|\hat{x}-\hat{b}\|} \rightarrow 1 \quad \text { as } \quad \varepsilon \downarrow 0 .
$$

By Eq. 76 and the above observations, letting $\varepsilon \downarrow 0$ implies $\operatorname{itr}[A, B](\bar{x}) \leq \alpha$ in view of Eq. 72.

We now $\operatorname{prove} \operatorname{itr}_{p}[A, B](\bar{x}) \leq \operatorname{itr}_{c}[A, B](\bar{x})$. Since the inequality becomes trivial when $\operatorname{itr}_{c}[A, B](\bar{x})=1$, we only need a proof for the case $\operatorname{itr}_{c}[A, B](\bar{x})<1$. We take an arbitrary number $\alpha$ satisfying $\operatorname{itr}_{c}[A, B](\bar{x})<\alpha<1$ and prove that $\operatorname{itr}_{p}[A, B](\bar{x}) \leq \alpha$. From Definition 4 , to obtain this inequality, it suffices to show that $\{A, B\}$ does not satisfy property $(\mathcal{P})$ at $\bar{x}$ with $\alpha$ (and any number $\varepsilon>0$ ).

Fix a number $\alpha_{1}$ satisfying $\operatorname{itr}_{c}[A, B](\bar{x})<\alpha_{1}<\alpha$. Since $2 \varepsilon(\alpha+1 / \sqrt{\varepsilon}) \downarrow 0$ as $\varepsilon \downarrow 0$ and $\alpha-\alpha_{1}>0$, there exists a number $\varepsilon_{0}>0$ such that $\left.\varepsilon \in\right] 0, \varepsilon_{0}$ [ is equivalent to $\varepsilon>0$ satisfying

$$
2 \varepsilon(\alpha+1 / \sqrt{\varepsilon})<\alpha-\alpha_{1} .
$$

We claim that for any $\varepsilon \in] 0, \varepsilon_{0}\left[\right.$, there exist $a \in(A \backslash B) \cap \mathbb{B}_{\varepsilon}(\bar{x}), b \in(B \backslash A) \cap \mathbb{B}_{\varepsilon}(\bar{x})$, $x \in \mathbb{B}_{\varepsilon}(\bar{x})$ with $\|x-a\|=\|x-b\|$ and a number $\delta>0$ such that Eq. 58 holds true for all $\rho \in] 0, \delta[$.

To prove the above claim, we first recall the following representation of $\operatorname{itr}_{c}[A, B](\bar{x})$ [32, Equation (15)]:

$$
\begin{aligned}
& \operatorname{itr}_{c}[A, B](\bar{x})=\quad \liminf _{a \rightarrow \bar{x}, b \rightarrow \bar{x}, x \rightarrow \bar{x}}\left\|v_{1}+v_{2}\right\|, \\
& a \in A \backslash B, b \in B \backslash A,\|x-a\|=\|x-b\| \\
& \operatorname{dist}\left(v_{1}, N_{A}(a)\right) \rightarrow 0, \operatorname{dist}\left(v_{2}, N_{B}(b)\right) \rightarrow 0,\left\|v_{1}\right\|+\left\|v_{2}\right\|=1
\end{aligned}
$$

with the convention that the infimum over the empty set equals 1 . 
In view of Eq. 78 and $\operatorname{itr}_{c}[A, B](\bar{x})<\alpha_{1}$, there exist $a \in(A \backslash B) \cap \mathbb{B}_{\varepsilon}(\bar{x}), b \in(B \backslash A) \cap$ $\mathbb{B}_{\varepsilon}(\bar{x}), x \in \mathbb{B}_{\varepsilon}(\bar{x})$ with $\|x-a\|=\|x-b\|$ and $v_{1}, v_{2} \in X$ satisfying

$$
\begin{gathered}
\operatorname{dist}\left(v_{1}, N_{A}(a)\right)<\varepsilon, \operatorname{dist}\left(v_{2}, N_{B}(b)\right)<\varepsilon,\left\|v_{1}\right\|+\left\|v_{2}\right\|=1,\left\|v_{1}+v_{2}\right\|<\alpha_{1}, \\
\left\langle v_{1}, x-a\right\rangle=\left\|v_{1}\right\|\|x-a\|,\left\langle v_{2}, x-b\right\rangle=\left\|v_{2}\right\|\|x-b\| .
\end{gathered}
$$

The last inequality in Eq. 79 and Eq. 77 imply $\left\|v_{1}+v_{2}\right\|+2 \varepsilon(\alpha+1 / \sqrt{\varepsilon})<\alpha$, which is Eq. 55.

Thus, the points $a, b, x$ together with the numbers $\alpha, \varepsilon>0$ and the vectors $v_{1}, v_{2}$ satisfy all the assumptions of Proposition 8. In view of this proposition, there exists a number $\delta>0$ such that Eq. 58 holds true for all $\rho \in] 0, \delta[$.

Hence, we have proven the above claim, which in turn implies that $\{A, B\}$ does not satisfy property $(\mathcal{P})$ at $\bar{x}$ with the number $\alpha$. This is because in view of Remark 9 , the statement that Eq. 58 holds true for all $\rho \in] 0, \delta[$ is the negation of the statement that there exists $\rho \in] 0, \delta\left[\right.$ such that Eq. 43 holds true. Then from Definition 4, we have $\operatorname{itr}_{p}[A, B](\bar{x}) \leq \alpha$.

The proof is complete.

The first estimate in Eq. 71 shows that property $(\mathcal{P})$ is a necessary condition for intrinsic transversality while the second one shows that it is a sufficient condition for the property characterized by the inequality $\operatorname{itr}_{c}[A, B](\bar{x})>0$. A combination of Lemma 2 and Theorem 1 eliminates the gap between these properties in the Hilbert space setting.

Theorem 2 (equivalences) Let $\{A, B\}$ be a pair of closed sets and $\bar{x} \in A \cap B$. Then the following statements hold true.

(i) If $\operatorname{itr}_{c}[A, B](\bar{x}) \geq 1 / \sqrt{2}$, then

$$
\min \left\{\operatorname{itr}[A, B](\bar{x}), \operatorname{itr}_{w}[A, B](\bar{x}), \operatorname{itr}_{p}[A, B](\bar{x}), \operatorname{itr}_{c}[A, B](\bar{x})\right\} \geq 1 / \sqrt{2} .
$$

(ii) If $\operatorname{itr}_{c}[A, B](\bar{x})<1 / \sqrt{2}$, then

$$
\operatorname{itr}[A, B](\bar{x})=\operatorname{itr}_{w}[A, B](\bar{x})=\operatorname{itr}_{p}[A, B](\bar{x})=\operatorname{itr}_{c}[A, B](\bar{x}) .
$$

Proof Item (i) follows from Theorem 1(i) and Eq. 71 while item (ii) follows from Theorem 1(ii) and Eq. 71.

The following observation is obvious in view of Theorem 2 .

Remark 10 (answer to Question 4) In Hilbert spaces, intrinsic transversality is equivalent to property $(\mathcal{P})$. Note that the latter does not involve elements of normal cones. Note also that in the case $\operatorname{itr}_{c}[A, B](\bar{x})>1 / \sqrt{2}$, the equality of $\operatorname{itr}_{p}[A, B](\bar{x})$ and $\operatorname{itr}[A, B](\bar{x})$ remains unknown.

\section{Intrinsic Transversality in Euclidean Spaces}

We first recall definitions of the relative limiting normals which are motivated by the compactness of the unit sphere in finite dimensional spaces as well as the fact that not all normal vectors are always involved in characterizing transversality-type properties. These notions were shown to be useful for analyzing intrinsic transversality and its variants. We refer the reader to [32, page 123] for a more thorough discussion. 
In this section, $X$ is a Euclidean space.

Definition 5 (relative limiting normal) [32, Definition 2] Let $A, B$ be closed sets and $\bar{x} \in A \cap B$.

(i) A pair $\left(v_{1}, v_{2}\right) \in X \times X$ is called a pair of relative limiting normals to $\{A, B\}$ at $\bar{x}$ if there exist sequences $\left(a_{k}\right) \subset A \backslash B,\left(b_{k}\right) \subset B \backslash A,\left(x_{k}\right) \subset X$ and $\left(v_{1 k}\right),\left(v_{2 k}\right) \subset X$ such that $a_{k} \rightarrow \bar{x}, b_{k} \rightarrow \bar{x}, x_{k} \rightarrow \bar{x}, v_{1 k} \rightarrow v_{1}, v_{2 k} \rightarrow v_{2}$, and

$$
\begin{gathered}
v_{1 k} \in N_{A}\left(a_{k}\right), v_{2 k} \in N_{B}\left(b_{k}\right) \quad(k=1,2, \ldots), \\
\frac{\left\|x_{k}-a_{k}\right\|}{\left\|x_{k}-b_{k}\right\|} \rightarrow 1, \frac{\left\langle v_{1 k}, x_{k}-a_{k}\right\rangle}{\left\|v_{1 k}\right\|\left\|x_{k}-a_{k}\right\|} \rightarrow 1, \frac{\left\langle v_{2 k}, x_{k}-b_{k}\right\rangle}{\left\|v_{2 k}\right\|\left\|x_{k}-b_{k}\right\|} \rightarrow 1,
\end{gathered}
$$

with the convention that $\frac{0}{0}=1$. The set of all pairs of relative limiting normals to $\{A, B\}$ at $\bar{x}$ is denoted by $\bar{N}_{A, B}(\bar{x})$.

(ii) A pair $\left(v_{1}, v_{2}\right) \in X \times X$ is called a pair of restricted relative limiting normals to $\{A, B\}$ at $\bar{x}$ if there exist sequences $\left(a_{k}\right) \subset A \backslash B,\left(b_{k}\right) \subset B \backslash A,\left(x_{k}\right) \subset X$ and $\left(v_{1 k}\right),\left(v_{2 k}\right) \subset X$ such that $\left\|x_{k}-a_{k}\right\|=\left\|x_{k}-b_{k}\right\|(k=1,2, \ldots), a_{k} \rightarrow \bar{x}, b_{k} \rightarrow \bar{x}$, $x_{k} \rightarrow \bar{x}, v_{1 k} \rightarrow v_{1}, v_{2 k} \rightarrow v_{2}$, and

$$
\begin{gathered}
\operatorname{dist}\left(v_{1 k}, N_{A}\left(a_{k}\right)\right) \rightarrow 0, \operatorname{dist}\left(v_{2 k}, N_{B}\left(b_{k}\right)\right) \rightarrow 0, \\
\left\langle v_{1 k}, x_{k}-a_{k}\right\rangle=\left\|v_{1 k}\right\|\left\|x_{k}-a_{k}\right\|,\left\langle v_{2 k}, x_{k}-b_{k}\right\rangle=\left\|v_{2 k}\right\|\left\|x_{k}-b_{k}\right\| \quad(k=1,2, \ldots) .
\end{gathered}
$$

The set of all pairs of restricted relative limiting normals to $\{A, B\}$ at $\bar{x}$ is denoted by $\bar{N}_{A, B}^{c}(\bar{x})$.

The following statement recalls a basic property of $\bar{N}_{A, B}(\bar{x})$ and $\bar{N}_{A, B}^{c}(\bar{x})$. In particular, they are cones. ${ }^{15}$

Proposition $9[32$, Proposition 2(i)] Let $A, B$ be closed sets and $\bar{x} \in A \cap B$. The sets $\bar{N}_{A, B}(\bar{x})$ and $\bar{N}_{A, B}^{c}(\bar{x})$ are closed cones in $X \times X$. Moreover, if $\left(v_{1}, v_{2}\right) \in \bar{N}_{A, B}(\bar{x})$ (respectively, $\bar{N}_{A, B}^{c}(\bar{x})$ ), then $\left(t_{1} v_{1}, t_{2} v_{2}\right) \in \bar{N}_{A, B}(\bar{x})$ (respectively, $\bar{N}_{A, B}^{c}(\bar{x})$ ) for all $t_{1}, t_{2}>0$.

The following equalities show the important role of the cones $\bar{N}_{A, B}(\bar{x})$ and $\bar{N}_{A, B}^{c}(\bar{x})$ in characterizing intrinsic transversality [32, Equations (19)\&(20)]:

$$
\begin{aligned}
\operatorname{itr}[A, B](\bar{x}) & \min _{\substack{\left(v_{1}, v_{2}\right) \in \bar{N}_{A, B}(\bar{x}) \\
\left\|v_{1}\right\|+\left\|v_{2}\right\|=1}}\left\|v_{1}+v_{2}\right\|, \\
\operatorname{itr}_{c}[A, B](\bar{x}) & =\min _{\substack{\left(v_{1}, v_{2}\right) \in \bar{N}_{A, B}^{c}(\bar{x}) \\
\left\|v_{1}\right\|+\left\|v_{2}\right\|=1}}\left\|v_{1}+v_{2}\right\|,
\end{aligned}
$$

with the convention that the minimum over the empty set equals 1 . Note that the minima in Eq. 80 and Eq. 81 are attainable thanks to the compactness of the constraint sets under the minima.

\footnotetext{
${ }^{15}$ Here, the empty set is viewed as a cone by convention.
} 
Remark 11 One can formulate a similar representation for $\operatorname{itr}_{w}[A, B](\bar{x})$ using another cone $\bar{N}_{A, B}^{w}(\bar{x})$ of 'weak' relative limiting normals satisfying

$$
\bar{N}_{A, B}^{c}(\bar{x}) \subset \bar{N}_{A, B}^{w}(\bar{x}) \subset \bar{N}_{A, B}(\bar{x}) .
$$

The goals of this section are 1) to establish a geometric counterpart of the analytic condition under which Theorem 1(ii) is valid, see Lemma 3; 2) to deduce new characterizations of intrinsic transversality in terms of relative limiting normals, see Theorem 3; and 3) to provide further insight into the quantitative results established in Section 2 when specialized to the Euclidean space setting, see Theorem 4. As by-products, we address the following questions raised by Kruger [32] regarding the cone objects appearing in Eq. 82, see Remarks 12 and 13.

Question 5 [32, question 4, page 140] What is the relationship between the two cones $\bar{N}_{A, B}(\bar{x})$ and $\bar{N}_{A, B}^{c}(\bar{x})$ ?

Question 6 [32, question 5, page 140] How can $\bar{N}_{A, B}^{w}(\bar{x})$ be used in characterizing intrinsic transversality?

Recall from Theorem 1 that if $\operatorname{itr}_{c}[A, B](\bar{x}) \geq 1 / \sqrt{2}$, then the intrinsic transversality property is satisfied with a quantitative constant at least $1 / \sqrt{2}$. This indicates that the other case, i.e.,

$$
\operatorname{itr}_{c}[A, B](\bar{x})<1 / \sqrt{2},
$$

is of the main interest in studying the property.

The analysis in this section is tuned to the scenario of Eq. 83. To begin, let us define the set:

$$
C:=\left\{\left(v_{1}, v_{2}\right) \in X \times X \mid\left\langle v_{1}, v_{2}\right\rangle<0\right\}
$$

Proposition 10 The set $C$ defined above is a cone in $X \times X$. Moreover, if $\left(v_{1}, v_{2}\right) \in C$, then $\left(t_{1} v_{1}, t_{2} v_{2}\right) \in C$ for all $t_{1}, t_{2}>0$.

Proof The proof is straightforward from the definition of $C$.

For convenience, we also define the set

$$
S:=\left\{\left(v_{1}, v_{2}\right) \in X \times X \mid\left\|v_{1}\right\|+\left\|v_{2}\right\|=1\right\},
$$

and note that $\bar{N}_{A, B}^{c}(\bar{x}) \cap S$ is the feasible set under the minimization in Eq. 81 .

The following result explains the role of $C$ in the analysis of intrinsic transversality in this paper.

Lemma 3 Let $A, B$ be closed sets and $\bar{x} \in A \cap B$. Then

$$
C \cap \bar{N}_{A, B}^{c}(\bar{x}) \cap S \neq \emptyset \quad \Leftrightarrow \quad \operatorname{itr}_{c}[A, B](\bar{x})<1 / \sqrt{2} .
$$

Proof $(\Rightarrow)$ We suppose $C \cap \bar{N}_{A, B}^{c}(\bar{x}) \cap S \neq \emptyset$ and prove that $\operatorname{itr}_{C}[A, B](\bar{x})<1 / \sqrt{2}$. Take a pair $\left(v_{1}, v_{2}\right) \in C \cap \bar{N}_{A, B}^{c}(\bar{x}) \cap S$. We note that $v_{1} \neq 0$ and $v_{2} \neq 0$ as $\left(v_{1}, v_{2}\right) \in C$, and set $v_{1}^{\prime}:=\frac{v_{1}}{2\left\|v_{1}\right\|}$ and $v_{2}^{\prime}:=\frac{v_{2}}{2\left\|v_{2}\right\|}$. It holds $\left(v_{1}^{\prime}, v_{2}^{\prime}\right) \in S$ since $\left\|v_{1}^{\prime}\right\|+\left\|v_{2}^{\prime}\right\|=1 / 2+1 / 2=1$. 
Thanks to Propositions 9 and 10, it also holds $\left(v_{1}^{\prime}, v_{2}^{\prime}\right) \in C \cap \bar{N}_{A, B}^{c}(\bar{x})$. Then we have in view of Eq. 81 that

$$
\operatorname{itr}_{c}[A, B](\bar{x})^{2}=\min _{\left(v_{1}, v_{2}\right) \in \bar{N}_{A, B}^{c}(\bar{x}) \cap S}\left\|v_{1}+v_{2}\right\|^{2} \leq\left\|v_{1}^{\prime}+v_{2}^{\prime}\right\|^{2}=1 / 2+2\left\langle v_{1}^{\prime}, v_{2}^{\prime}\right\rangle<1 / 2,
$$

where the last inequality holds true due to Eq. 84 as $\left(v_{1}^{\prime}, v_{2}^{\prime}\right) \in C$. Hence $\operatorname{itr}_{c}[A, B](\bar{x})<$ $1 / \sqrt{2}$.

$(\Leftarrow)$ We suppose $C \cap \bar{N}_{A, B}^{c}(\bar{x}) \cap S=\emptyset$ and $\operatorname{prove}$ that $\operatorname{itr}_{c}[A, B](\bar{x}) \geq 1 / \sqrt{2}$. If $\bar{N}_{A, B}^{c}(\bar{x}) \cap S=\emptyset$, then Eq. 81 yields $\operatorname{itr}_{c}[A, B](\bar{x})=1>1 / \sqrt{2}$. We consider the case $\bar{N}_{A, B}^{c}(\bar{x}) \cap S \neq \emptyset$. Take an arbitrary pair $\left(v_{1}, v_{2}\right) \in \bar{N}_{A, B}^{c}(\bar{x}) \cap S$. Then by the assumption, it holds $\left(v_{1}, v_{2}\right) \notin C$, i.e., $\left\langle v_{1}, v_{2}\right\rangle \geq 0$. This implies

$\left\|v_{1}+v_{2}\right\|^{2}=\left\|v_{1}\right\|^{2}+\left\|v_{2}\right\|^{2}+2\left\langle v_{1}, v_{2}\right\rangle \geq\left\|v_{1}\right\|^{2}+\left\|v_{2}\right\|^{2} \geq \frac{1}{2}\left(\left\|v_{1}\right\|+\left\|v_{2}\right\|\right)^{2}=1 / 2$.

We have just proved that

$$
\left\|v_{1}+v_{2}\right\| \geq 1 / \sqrt{2} \text { for all }\left(v_{1}, v_{2}\right) \in \bar{N}_{A, B}^{c}(\bar{x}) \cap S .
$$

This together with the equality Eq. 81 implies that $\operatorname{itr}_{c}[A, B](\bar{x}) \geq 1 / \sqrt{2}$.

Lemma 3 provides an intuitive geometric counterpart of the quite mysterious analytic condition Eq. 83. It also gives rise to the following characterizations of intrinsic transversality.

Theorem 3 (refined characterizations of intrinsic transversality) Let $A, B$ be closed sets and $\bar{x} \in A \cap B$. Then the following statements are equivalent.

(i) $\{A, B\}$ is intrinsically transversal at $\bar{x}$.

(ii) There exists a number $\alpha \in] 0,1[$ such that

$$
\left\|v_{1}+v_{2}\right\|>\alpha \quad \text { for all }\left(v_{1}, v_{2}\right) \in C \cap \bar{N}_{A, B}^{c}(\bar{x}) \cap S .
$$

(iii) $\left\{v \in X \mid(v,-v) \in C \cap \bar{N}_{A, B}^{c}(\bar{x})\right\} \subset\{0\}$.

Proof (i) $\Rightarrow$ (ii). We suppose that $\{A, B\}$ is intrinsically transversal at $\bar{x}$. This is equiva-

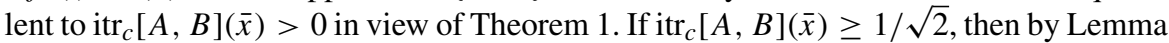
3 the intersection $C \cap \bar{N}_{A, B}^{c}(\bar{x}) \cap S$ is empty, and hence the conclusion is trivial. We analyze the case $\operatorname{itr}_{c}[A, B](\bar{x})<1 / \sqrt{2}$. Take any $\alpha$ satisfying $0<\alpha<\operatorname{itr}_{c}[A, B](\bar{x})$. Then in view of Eq. 81 we have that

$$
\left\|v_{1}+v_{2}\right\| \geq \operatorname{itr}_{c}[A, B](\bar{x})>\alpha \quad \text { for all } \quad\left(v_{1}, v_{2}\right) \in \bar{N}_{A, B}^{c}(\bar{x}) \cap S,
$$

which obviously implies Eq. 85.

(ii) $\Rightarrow$ (iii). Suppose that (iii) is violated, i.e., there exists $v \neq 0$ such that $(v,-v) \in C \cap$ $\bar{N}_{A, B}^{c}(\bar{x})$. Then the pair $\left(v_{1}, v_{2}\right):=\left(\frac{v}{2\|v\|},-\frac{v}{2\|v\|}\right) \in C \cap \bar{N}_{A, B}^{c}(\bar{x}) \cap S$, but $\left\|v_{1}+v_{2}\right\|=0$. That is, (ii) is violated.

(iii) $\Rightarrow$ (i). Suppose that (i) is violated, i.e., $\operatorname{itr}_{c}[A, B](\bar{x})=0$. By [32, Corollary 3, (i) $\Leftrightarrow($ ii) $]$, there exists a pair of vectors $\left(v_{1}, v_{2}\right) \in \bar{N}_{A, B}^{c}(\bar{x}) \cap S$ satisfying $\left\|v_{1}+v_{2}\right\|=0$. The latter conditions trivially imply $v_{1}=-v_{2} \neq 0$ and $\left\langle v_{1}, v_{2}\right\rangle<0$. Then the vector $v \neq 0$ satisfies $(v,-v):=\left(v_{1}, v_{2}\right) \in C \cap \bar{N}_{A, B}^{c}(\bar{x})$. That is, (iii) is violated. 
An implication of Theorem 3 is that only pairs of relative limiting normals in $C$ are relevant for characterizing intrinsic transversality. This observation guides us to the following concrete relationship between the two cones $\bar{N}_{A, B}(\bar{x})$ and $\bar{N}_{A, B}^{c}(\bar{x})$.

Theorem 4 Let $A, B$ be closed sets and $\bar{x} \in A \cap B$. Then

$$
\bar{N}_{A, B}^{c}(\bar{x}) \cap C=\bar{N}_{A, B}(\bar{x}) \cap C .
$$

Proof It is known by [32, Proposition 2(ii)] that $\bar{N}_{A, B}^{c}(\bar{x}) \subset \bar{N}_{A, B}(\bar{x})$. Thus, it is sufficient to show that

$$
\bar{N}_{A, B}(\bar{x}) \cap C \subset \bar{N}_{A, B}^{c}(\bar{x}) \cap C .
$$

Let us take any $\left(v_{1}, v_{2}\right) \in \bar{N}_{A, B}(\bar{x}) \cap C$. Then by the definition of $\bar{N}_{A, B}(\bar{x})$, there exist sequences $\left(a_{k}\right) \subset A \backslash B,\left(b_{k}\right) \subset B \backslash A,\left(x_{k}\right) \subset X$ and $\left(v_{1 k}\right),\left(v_{2 k}\right) \subset X$ such that $a_{k} \rightarrow \bar{x}$, $b_{k} \rightarrow \bar{x}, x_{k} \rightarrow \bar{x}, v_{1 k} \rightarrow v_{1}, v_{2 k} \rightarrow v_{2}$ and

$$
\begin{gathered}
v_{1 k} \in N_{A}\left(a_{k}\right), v_{2 k} \in N_{B}\left(b_{k}\right) \quad(k=1,2, \ldots), \\
\frac{\left\|x_{k}-a_{k}\right\|}{\left\|x_{k}-b_{k}\right\|} \rightarrow 1, \frac{\left\langle v_{1 k}, x_{k}-a_{k}\right\rangle}{\left\|v_{1 k}\right\|\left\|x_{k}-a_{k}\right\|} \rightarrow 1, \frac{\left\langle v_{2 k}, x_{k}-b_{k}\right\rangle}{\left\|v_{1 k}\right\|\left\|x_{k}-b_{k}\right\|} \rightarrow 1 .
\end{gathered}
$$

Note that $v_{1} \neq 0$ and $v_{2} \neq 0$ as $\left(v_{1}, v_{2}\right) \in C$. Then thanks to $v_{1 k} \rightarrow v_{1}$ and $v_{2 k} \rightarrow v_{2}$, we can assume that $v_{1 k} \neq 0$ and $v_{2 k} \neq 0$ for all $k \in \mathbb{N}$. In view of Remark 2, we can also assume that $x_{k} \neq a_{k}$ and $x_{k} \neq b_{k}$ for all $k \in \mathbb{N}$.

Since $\left(v_{1}, v_{2}\right) \in C$, it holds that $\left\langle v_{1}, v_{2}\right\rangle<0$, equivalently,

$$
\left\|\frac{v_{1}}{\left\|v_{1}\right\|}-\frac{v_{2}}{\left\|v_{2}\right\|}\right\|>\sqrt{2} .
$$

To complete the proof, it suffices to prove that $\left(v_{1}, v_{2}\right) \in \bar{N}_{A, B}^{c}(\bar{x})$. For each $k=1,2, \ldots$, let us define:

$$
\begin{gathered}
m_{k}:=\frac{a_{k}+b_{k}}{2}, \\
x_{k}^{\prime}:=x_{k}-\frac{\left\langle b_{k}-a_{k}, x_{k}-m_{k}\right\rangle}{\left\|b_{k}-a_{k}\right\|^{2}}\left(b_{k}-a_{k}\right), \\
v_{1 k}^{\prime}:=\frac{\left\|v_{1 k}\right\|}{\left\|x_{k}^{\prime}-a_{k}\right\|}\left(x_{k}^{\prime}-a_{k}\right), v_{2 k}^{\prime}:=\frac{\left\|v_{2 k}\right\|}{\left\|x_{k}^{\prime}-b_{k}\right\|}\left(x_{k}^{\prime}-b_{k}\right) .
\end{gathered}
$$

All we need is to verify the following four conditions:

$$
\begin{gathered}
\left\|x_{k}^{\prime}-a_{k}\right\|=\left\|x_{k}^{\prime}-b_{k}\right\| \quad(k=1,2, \ldots), \\
x_{k}^{\prime} \rightarrow \bar{x}, v_{1 k}^{\prime} \rightarrow v_{1}, v_{2 k}^{\prime} \rightarrow v_{2} \quad(k=1,2, \ldots), \\
\operatorname{dist}\left(v_{1 k}^{\prime}, N_{A}\left(a_{k}\right)\right) \rightarrow 0, \operatorname{dist}\left(v_{2 k}^{\prime}, N_{B}\left(b_{k}\right)\right) \rightarrow 0, \\
\left\langle v_{1 k}^{\prime}, x_{k}^{\prime}-a_{k}\right\rangle=\left\|v_{1 k}^{\prime}\right\|\left\|x_{k}^{\prime}-a_{k}\right\|,\left\langle v_{2 k}^{\prime}, x_{k}^{\prime}-b_{k}\right\rangle=\left\|v_{2 k}^{\prime}\right\|\left\|x_{k}^{\prime}-b_{k}\right\| .
\end{gathered}
$$

Condition Eq. 91. This follows from Eq. 89 since for each $k=1,2, \ldots$, we have that

$$
\begin{aligned}
\left\|x_{k}^{\prime}-a_{k}\right\|^{2}-\left\|x_{k}^{\prime}-b_{k}\right\|^{2} & =\left\|x_{k}-a_{k}\right\|^{2}-\left\|x_{k}-b_{k}\right\|^{2}-2 \frac{\left\langle b_{k}-a_{k}, x_{k}-m_{k}\right\rangle}{\left\|b_{k}-a_{k}\right\|^{2}}\left\langle x_{k}-a_{k}, b_{k}-a_{k}\right\rangle \\
& +2 \frac{\left\langle b_{k}-a_{k}, x_{k}-m_{k}\right\rangle}{\left\|b_{k}-a_{k}\right\|^{2}}\left\langle x_{k}-b_{k}, b_{k}-a_{k}\right\rangle \\
& =\left\|x_{k}-a_{k}\right\|^{2}-\left\|x_{k}-b_{k}\right\|^{2}-2 \frac{\left\langle b_{k}-a_{k}, x_{k}-m_{k}\right\rangle}{\left\|b_{k}-a_{k}\right\|^{2}}\left\langle b_{k}-a_{k}, b_{k}-a_{k}\right\rangle \\
& =\left\|x_{k}-a_{k}\right\|^{2}-\left\|x_{k}-b_{k}\right\|^{2}-2\left\langle b_{k}-a_{k}, x_{k}-m_{k}\right\rangle \\
& =\left\|x_{k}-a_{k}\right\|^{2}-\left\|x_{k}-b_{k}\right\|^{2}-\left\langle\left(x_{k}-a_{k}\right)-\left(x_{k}-b_{k}\right),\left(x_{k}-a_{k}\right)+\left(x_{k}-b_{k}\right)\right\rangle=0 .
\end{aligned}
$$


Condition Eq. 94. We first infer from Eq. 91 and $a_{k} \neq b_{k}$ that $x_{k}^{\prime} \neq a_{k}$ and $x_{k}^{\prime} \neq b_{k}$. This ensures that $v_{1 k}^{\prime}$ and $v_{2 k}^{\prime}$ in Eq. 90 are well defined. Then thanks to Eq. 90, we have that

$$
\begin{aligned}
\left\|v_{1 k}^{\prime}\right\|=\left\|v_{1 k}\right\|,\left\|v_{2 k}^{\prime}\right\| & =\left\|v_{2 k}\right\|, \\
\left\langle v_{1 k}^{\prime}, x_{k}^{\prime}-a_{k}\right\rangle=\left\|v_{1 k}\right\|\left\|x_{k}^{\prime}-a_{k}\right\| & =\left\|v_{1 k}^{\prime}\right\|\left\|x_{k}^{\prime}-a_{k}\right\|, \\
\left\langle v_{2 k}^{\prime}, x_{k}^{\prime}-b_{k}\right\rangle=\left\|v_{2 k}\right\|\left\|x_{k}^{\prime}-b_{k}\right\| & =\left\|v_{2 k}^{\prime}\right\|\left\|x_{k}^{\prime}-b_{k}\right\| .
\end{aligned}
$$

Condition Eq. 93. Since $v_{1 k} \in N_{A}\left(a_{k}\right)$ and $v_{2 k} \in N_{A}\left(b_{k}\right)(k=1,2, \ldots)$, whenever condition Eq. 92 has been verified, we have that

$$
\begin{aligned}
& \operatorname{dist}\left(v_{1 k}^{\prime}, N_{A}\left(a_{k}\right)\right) \leq\left\|v_{1 k}^{\prime}-v_{1 k}\right\| \leq\left\|v_{1 k}^{\prime}-v_{1}\right\|+\left\|v_{1 k}-v_{1}\right\| \rightarrow 0, \\
& \operatorname{dist}\left(v_{2 k}^{\prime}, N_{B}\left(b_{k}\right)\right) \leq\left\|v_{2 k}^{\prime}-v_{2 k}\right\| \leq\left\|v_{2 k}^{\prime}-v_{2}\right\|+\left\|v_{2 k}-v_{2}\right\| \rightarrow 0 .
\end{aligned}
$$

Condition Eq. 92. Since $x_{k} \rightarrow \bar{x}, a_{k} \rightarrow \bar{x}$ and $b_{k} \rightarrow \bar{x}$, it holds by Eq. 89 that

$$
\left\|x_{k}^{\prime}-x_{k}\right\|=\left\|\frac{\left\langle b_{k}-a_{k}, x_{k}-m_{k}\right\rangle}{\left\|b_{k}-a_{k}\right\|^{2}}\left(b_{k}-a_{k}\right)\right\| \leq\left\|x_{k}-m_{k}\right\|=\left\|x_{k}-\frac{a_{k}+b_{k}}{2}\right\| \rightarrow 0 .
$$

Then

$$
\left\|x_{k}^{\prime}-\bar{x}\right\| \leq\left\|x_{k}^{\prime}-x_{k}\right\|+\left\|x_{k}-\bar{x}\right\| \rightarrow 0 .
$$

In the remainder of the proof, we show that $v_{1 k}^{\prime} \rightarrow v_{1}$ while the condition $v_{2 k}^{\prime} \rightarrow v_{2}$ is obtained in a similar manner. Since $v_{1 k} \rightarrow v_{1}$, all we need is to show that $\left\|v_{1 k}^{\prime}-v_{1 k}\right\| \rightarrow 0$. Note that by Eq. 90 it holds

$$
\begin{aligned}
\left\|v_{1 k}^{\prime}-v_{1 k}\right\| & =\left\|\frac{\left\|v_{1 k}\right\|}{\left\|x_{k}^{\prime}-a_{k}\right\|}\left(x_{k}^{\prime}-a_{k}\right)-v_{1 k}\right\|=\left\|v_{1 k}\right\|\left\|\frac{x_{k}^{\prime}-a_{k}}{\left\|x_{k}^{\prime}-a_{k}\right\|}-\frac{v_{1 k}}{\left\|v_{1 k}\right\|}\right\| \\
& \leq\left\|v_{1 k}\right\|\left(\left\|\frac{x_{k}-a_{k}}{\left\|x_{k}-a_{k}\right\|}-\frac{v_{1 k}}{\left\|v_{1 k}\right\|}\right\|+\left\|\frac{x_{k}^{\prime}-a_{k}}{\left\|x_{k}^{\prime}-a_{k}\right\|}-\frac{x_{k}-a_{k}}{\left\|x_{k}-a_{k}\right\|}\right\|\right) .
\end{aligned}
$$

Note also that due to Eq. 87,

$$
\left\|\frac{x_{k}-a_{k}}{\left\|x_{k}-a_{k}\right\|}-\frac{v_{1 k}}{\left\|v_{1 k}\right\|}\right\|=\sqrt{2-2 \frac{\left\langle v_{1 k}, x_{k}-a_{k}\right\rangle}{\left\|v_{1 k}\right\|\left\|x_{k}-a_{k}\right\|}} \rightarrow 0 .
$$

In view of Eq. 95 and Eq. 96, in order to obtain $\left\|v_{1 k}^{\prime}-v_{1 k}\right\| \rightarrow 0$, it suffices to prove that

$$
\left\|\frac{x_{k}^{\prime}-a_{k}}{\left\|x_{k}^{\prime}-a_{k}\right\|}-\frac{x_{k}-a_{k}}{\left\|x_{k}-a_{k}\right\|}\right\| \rightarrow 0 .
$$

To proceed, let us take any number $\varepsilon>0$ which can be arbitrarily small and show the existence of a natural $N \in \mathbb{N}$ such that

$$
\left\|\frac{x_{k}^{\prime}-a_{k}}{\left\|x_{k}^{\prime}-a_{k}\right\|}-\frac{x_{k}-a_{k}}{\left\|x_{k}-a_{k}\right\|}\right\|<\varepsilon, \quad \forall k \geq N .
$$

Choose a number $\varepsilon^{\prime}>0$ and satisfying

$$
2 \sqrt{\frac{2 \varepsilon^{\prime}-\varepsilon^{\prime 2}}{4-6 \varepsilon^{\prime}+3 \varepsilon^{\prime 2}}}<\varepsilon
$$

Such a number $\varepsilon^{\prime}$ exists since $\varepsilon>0$ and $\lim _{t \downarrow 0} 2 \sqrt{\frac{2 t-t^{2}}{4-6 t+3 t^{2}}}=0$. 
By the convergence conditions in Eq. 87, there exists a natural number $N \in \mathbb{N}$ such that $\forall k \geq N$,

$$
\begin{gathered}
1-\varepsilon^{\prime}<\frac{\left\|x_{k}-a_{k}\right\|}{\left\|x_{k}-b_{k}\right\|}<1+\varepsilon^{\prime}, \\
\frac{\left\langle v_{1 k}, x_{k}-a_{k}\right\rangle}{\left\|v_{1 k}\right\|\left\|x_{k}-a_{k}\right\|}>1-\varepsilon^{\prime}, \frac{\left\langle v_{2 k}, x_{k}-b_{k}\right\rangle}{\left\|v_{1 k}\right\|\left\|x_{k}-b_{k}\right\|}>1-\varepsilon^{\prime} .
\end{gathered}
$$

The estimates in Eq. 100 amount to

$$
\left\|\frac{v_{1 k}}{\left\|v_{1 k}\right\|}-\frac{x_{k}-a_{k}}{\left\|x_{k}-a_{k}\right\|}\right\|<\sqrt{2 \varepsilon^{\prime}},\left\|\frac{v_{2 k}}{\left\|v_{2 k}\right\|}-\frac{x_{k}-b_{k}}{\left\|x_{k}-b_{k}\right\|}\right\|<\sqrt{2 \varepsilon^{\prime}} .
$$

In order to prove Eq. 97, we first note that

$$
\left\langle x_{k}-x_{k}^{\prime}, x_{k}^{\prime}-m_{k}\right\rangle=0 .
$$

Indeed, by Eq. 89, it holds that

$$
\left\langle x_{k}-x_{k}^{\prime}, x_{k}^{\prime}-m_{k}\right\rangle=\frac{\left\langle b_{k}-a_{k}, x_{k}-m_{k}\right\rangle}{\left\|b_{k}-a_{k}\right\|^{2}}\left\langle b_{k}-a_{k}, x_{k}^{\prime}-m_{k}\right\rangle,
$$

from which Eq. 102 follows since

$$
\begin{aligned}
\left\langle b_{k}-a_{k}, x_{k}^{\prime}-m_{k}\right\rangle & =\left\langle b_{k}-a_{k}, x_{k}-m_{k}-\frac{\left\langle b_{k}-a_{k}, x_{k}-m_{k}\right\rangle}{\left\|b_{k}-a_{k}\right\|^{2}}\left(b_{k}-a_{k}\right)\right\rangle \\
& =\left\langle b_{k}-a_{k}, x_{k}-m_{k}\right\rangle-\frac{\left\langle b_{k}-a_{k}, x_{k}-m_{k}\right\rangle}{\left\|b_{k}-a_{k}\right\|^{2}}\left\langle b_{k}-a_{k}, b_{k}-a_{k}\right\rangle=0 .
\end{aligned}
$$

Second, we show that $\forall k \geq N$,

$$
\left\|x_{k}-x_{k}^{\prime}\right\|^{2} \leq \frac{2 \varepsilon^{\prime}-\varepsilon^{\prime 2}}{4\left(1-\varepsilon^{\prime}\right)^{2}} \min \left\{\left\|x_{k}-a_{k}\right\|^{2},\left\|x_{k}-b_{k}\right\|^{2}\right\} .
$$

If $\left\|x_{k}-a_{k}\right\| \geq\left\|x_{k}-b_{k}\right\|$, then

$$
\left\langle b_{k}-a_{k}, x_{k}-m_{k}\right\rangle \geq 0 .
$$

Note from Eq. 88 and Eq. 101 that

$$
\begin{gathered}
\left\|\frac{x_{k}-a_{k}}{\left\|x_{k}-a_{k}\right\|}-\frac{x_{k}-b_{k}}{\left\|x_{k}-b_{k}\right\|}\right\| \geq\left\|\frac{v_{1 k}}{\left\|v_{1 k}\right\|}-\frac{v_{2 k}}{\left\|v_{2 k}\right\|}\right\|-\left\|\frac{v_{1 k}}{\left\|v_{1 k}\right\|}-\frac{x_{k}-a_{k}}{\left\|x_{k}-a_{k}\right\|}\right\|-\left\|\frac{v_{2 k}}{\left\|v_{2 k}\right\|}-\frac{x_{k}-b_{k}}{\left\|x_{k}-b_{k}\right\|}\right\| \\
>\sqrt{2}-2 \sqrt{2 \varepsilon^{\prime}} .
\end{gathered}
$$

This, combined with Eq. 99 yields that

$$
\begin{aligned}
\left\langle x_{k}-a_{k}, x_{k}-b_{k}\right\rangle & <4\left(\sqrt{\varepsilon^{\prime}}-\varepsilon^{\prime}\right)\left\|x_{k}-a_{k}\right\|\left\|x_{k}-b_{k}\right\| \\
& <4\left(\sqrt{\varepsilon^{\prime}}-\varepsilon^{\prime}\right)\left(1+\varepsilon^{\prime}\right)\left\|x_{k}-b_{k}\right\|^{2} .
\end{aligned}
$$

Then

$$
\begin{aligned}
\left\langle b_{k}-a_{k}, x_{k}-b_{k}\right\rangle & =\left\langle b_{k}-x_{k}, x_{k}-b_{k}\right\rangle+\left\langle x_{k}-a_{k}, x_{k}-b_{k}\right\rangle \\
& =-\left\|x_{k}-b_{k}\right\|^{2}+\left\langle x_{k}-a_{k}, x_{k}-b_{k}\right\rangle \\
& <-\left\|x_{k}-b_{k}\right\|^{2}+4\left(\sqrt{\varepsilon^{\prime}}-\varepsilon^{\prime}\right)\left(1+\varepsilon^{\prime}\right)\left\|x_{k}-b_{k}\right\|^{2} \\
& =-\left(1-4\left(\sqrt{\varepsilon^{\prime}}-\varepsilon^{\prime}\right)\left(1+\varepsilon^{\prime}\right)\right)\left\|x_{k}-b_{k}\right\|^{2}<0 .
\end{aligned}
$$


From Eq. 89, Eq. 104 and Eq. 105 we have that

$$
\begin{aligned}
\left\|x_{k}^{\prime}-b_{k}\right\|^{2}-\left\|x_{k}-b_{k}\right\|^{2} & =\left\|x_{k}^{\prime}-x_{k}\right\|^{2}+2\left\langle x_{k}^{\prime}-x_{k}, x_{k}-b_{k}\right\rangle \\
& =\left\|x_{k}^{\prime}-x_{k}\right\|^{2}-2 \frac{\left\langle b_{k}-a_{k}, x_{k}-m_{k}\right\rangle}{\left\|b_{k}-a_{k}\right\|^{2}}\left\langle b_{k}-a_{k}, x_{k}-b_{k}\right\rangle \\
& \geq\left\|x_{k}^{\prime}-x_{k}\right\|^{2} .
\end{aligned}
$$

By Eq. 91 and Eq. 102 we get that

$$
\begin{aligned}
\left\|x_{k}-a_{k}\right\|^{2}+\left\|x_{k}-b_{k}\right\|^{2} & =2\left\|x_{k}-x_{k}^{\prime}\right\|^{2}+\left\|x_{k}^{\prime}-a_{k}\right\|^{2}+\left\|x_{k}^{\prime}-b_{k}\right\|^{2}+2\left\langle x_{k}-x_{k}^{\prime}, 2 x_{k}^{\prime}-\left(a_{k}+b_{k}\right)\right\rangle \\
& =2\left\|x_{k}-x_{k}^{\prime}\right\|^{2}+2\left\|x_{k}^{\prime}-b_{k}\right\|^{2}+4\left\langle x_{k}-x_{k}^{\prime}, x_{k}^{\prime}-m_{k}\right\rangle \\
& =2\left\|x_{k}-x_{k}^{\prime}\right\|^{2}+2\left\|x_{k}^{\prime}-b_{k}\right\|^{2} .
\end{aligned}
$$

This together with Eq. 99 and Eq. 106 yields

$$
\begin{aligned}
2\left\|x_{k}-x_{k}^{\prime}\right\|^{2} & =\left\|x_{k}-a_{k}\right\|^{2}+\left\|x_{k}-b_{k}\right\|^{2}-2\left\|x_{k}^{\prime}-b_{k}\right\|^{2} \\
& \leq\left(1+\varepsilon^{\prime}\right)^{2}\left\|x_{k}-b_{k}\right\|^{2}+\left\|x_{k}-b_{k}\right\|^{2}-2\left\|x_{k}^{\prime}-b_{k}\right\|^{2} \\
& \leq\left(1+\varepsilon^{\prime}\right)^{2}\left\|x_{k}-b_{k}\right\|^{2}+\left\|x_{k}-b_{k}\right\|^{2}-2\left(\left\|x_{k}-b_{k}\right\|^{2}+\left\|x_{k}^{\prime}-x_{k}\right\|^{2}\right) .
\end{aligned}
$$

Hence

$$
4\left\|x_{k}-x_{k}^{\prime}\right\|^{2} \leq\left(2 \varepsilon^{\prime}+\varepsilon^{\prime 2}\right)\left\|x_{k}-b_{k}\right\|^{2}=\left(2 \varepsilon^{\prime}+\varepsilon^{\prime 2}\right) \min \left\{\left\|x_{k}-a_{k}\right\|^{2},\left\|x_{k}-b_{k}\right\|^{2}\right\}
$$

since $\left\|x_{k}-a_{k}\right\| \geq\left\|x_{k}-b_{k}\right\|$ in this case.

By a similar argument, if $\left\|x_{k}-a_{k}\right\| \leq\left\|x_{k}-b_{k}\right\|$, then

$$
\left\langle b_{k}-a_{k}, x_{k}-m_{k}\right\rangle \leq 0,\left\langle b_{k}-a_{k}, x_{k}-a_{k}\right\rangle \geq 0 .
$$

Thus

$$
\begin{aligned}
\left\|x_{k}^{\prime}-a_{k}\right\|^{2}-\left\|x_{k}-a_{k}\right\|^{2} & =\left\|x_{k}^{\prime}-x_{k}\right\|^{2}+2\left\langle x_{k}^{\prime}-x_{k}, x_{k}-a_{k}\right\rangle \\
& =\left\|x_{k}^{\prime}-x_{k}\right\|^{2}-2 \frac{\left\langle b_{k}-a_{k}, x_{k}-m_{k}\right\rangle}{\left\|b_{k}-a_{k}\right\|^{2}}\left\langle b_{k}-a_{k}, x_{k}-a_{k}\right\rangle \\
& \geq\left\|x_{k}^{\prime}-x_{k}\right\|^{2} .
\end{aligned}
$$

By Eq. 91 and Eq. 102 we get that

$$
\left\|x_{k}-a_{k}\right\|^{2}+\left\|x_{k}-b_{k}\right\|^{2}=2\left\|x_{k}-x_{k}^{\prime}\right\|^{2}+2\left\|x_{k}^{\prime}-a_{k}\right\|^{2},
$$

which together with Eq. 99 and Eq. 108 yields that

$$
2\left\|x_{k}-x_{k}^{\prime}\right\|^{2} \leq\left\|x_{k}-a_{k}\right\|^{2}+\frac{1}{\left(1-\varepsilon^{\prime}\right)^{2}}\left\|x_{k}-a_{k}\right\|^{2}-2\left(\left\|x_{k}-a_{k}\right\|^{2}+\left\|x_{k}^{\prime}-x_{k}\right\|^{2}\right) .
$$

Equivalently,

$$
4\left\|x_{k}-x_{k}^{\prime}\right\|^{2} \leq \frac{2 \varepsilon^{\prime}-\varepsilon^{\prime 2}}{\left(1-\varepsilon^{\prime}\right)^{2}}\left\|x_{k}-a_{k}\right\|^{2}=\frac{2 \varepsilon^{\prime}-\varepsilon^{\prime 2}}{\left(1-\varepsilon^{\prime}\right)^{2}} \min \left\{\left\|x_{k}-a_{k}\right\|^{2},\left\|x_{k}-b_{k}\right\|^{2}\right\}
$$

since $\left\|x_{k}-a_{k}\right\| \leq\left\|x_{k}-b_{k}\right\|$ in this case.

Combining Eq. 107 and Eq. 109 and noting that $2 \varepsilon^{\prime}+\varepsilon^{\prime 2}<\frac{2 \varepsilon^{\prime}-\varepsilon^{\prime 2}}{\left(1-\varepsilon^{\prime}\right)^{2}}$, we obtain Eq. 103 as claimed.

Third, we show that $\forall k \geq N$

$$
\left\|x_{k}^{\prime}-a_{k}\right\|^{2} \geq \frac{4-6 \varepsilon^{\prime}+3 \varepsilon^{\prime 2}}{2 \varepsilon^{\prime}-\varepsilon^{\prime 2}}\left\|x_{k}-x_{k}^{\prime}\right\|^{2} .
$$


Indeed, if $\left\|x_{k}-a_{k}\right\| \leq\left\|x_{k}-b_{k}\right\|$, then the use of Eq. 108 and Eq. 109 yields Eq. 110:

$$
\begin{aligned}
\left\|x_{k}^{\prime}-a_{k}\right\|^{2} & \geq\left\|x_{k}-a_{k}\right\|^{2}+\left\|x_{k}-x_{k}^{\prime}\right\|^{2} \\
& \geq \frac{\left.4\left(1-\varepsilon^{\prime}\right)^{2}\right)}{2 \varepsilon^{\prime}-\varepsilon^{\prime 2}}\left\|x_{k}-x_{k}^{\prime}\right\|^{2}+\left\|x_{k}-x_{k}^{\prime}\right\|^{2}=\frac{4-6 \varepsilon^{\prime}+3 \varepsilon^{\prime 2}}{2 \varepsilon^{\prime}-\varepsilon^{\prime 2}}\left\|x_{k}-x_{k}^{\prime}\right\|^{2} .
\end{aligned}
$$

Otherwise, i.e., if $\left\|x_{k}-a_{k}\right\| \geq\left\|x_{k}-b_{k}\right\|$, then the successive use of Eq. 91, Eq. 106 and Eq. 103 implies that

$$
\begin{gathered}
\left\|x_{k}^{\prime}-a_{k}\right\|^{2}=\left\|x_{k}^{\prime}-b_{k}\right\|^{2} \geq\left\|x_{k}-b_{k}\right\|^{2}+\left\|x_{k}-x_{k}^{\prime}\right\|^{2} \\
\geq \frac{4}{2 \varepsilon^{\prime}+\varepsilon^{\prime 2}}\left\|x_{k}-x_{k}^{\prime}\right\|^{2}+\left\|x_{k}^{\prime}-x_{k}\right\|^{2}=\frac{4+2 \varepsilon^{\prime}+\varepsilon^{\prime 2}}{2 \varepsilon^{\prime}+\varepsilon^{\prime 2}}\left\|x_{k}-x_{k}^{\prime}\right\|^{2},
\end{gathered}
$$

which also yields Eq. 110 since $\frac{4+2 \varepsilon^{\prime}+\varepsilon^{\prime 2}}{2 \varepsilon^{\prime}+\varepsilon^{\prime 2}}>\frac{4-6 \varepsilon^{\prime}+3 \varepsilon^{\prime 2}}{2 \varepsilon^{\prime}-\varepsilon^{\prime 2}}$. Hence Eq. 110 has been proved.

Fourth, we show that $\forall k \geq N$

$$
\left\|\frac{x_{k}^{\prime}-a_{k}}{\left\|x_{k}^{\prime}-a_{k}\right\|}-\frac{x_{k}-a_{k}}{\left\|x_{k}-a_{k}\right\|}\right\| \leq 2 \frac{\left\|x_{k}-x_{k}^{\prime}\right\|}{\left\|x_{k}^{\prime}-a_{k}\right\|} .
$$

Indeed,

$$
\begin{aligned}
\left\|\frac{x_{k}^{\prime}-a_{k}}{\left\|x_{k}^{\prime}-a_{k}\right\|}-\frac{x_{k}-a_{k}}{\left\|x_{k}-a_{k}\right\|}\right\| & \leq\left\|\frac{x_{k}^{\prime}-a_{k}}{\left\|x_{k}^{\prime}-a_{k}\right\|}-\frac{x_{k}-a_{k}}{\left\|x_{k}^{\prime}-a_{k}\right\|}\right\|+\left\|\frac{x_{k}-a_{k}}{\left\|x_{k}^{\prime}-a_{k}\right\|}-\frac{x_{k}-a_{k}}{\left\|x_{k}-a_{k}\right\|}\right\| \\
& =\frac{\left\|x_{k}-x_{k}^{\prime}\right\|}{\left\|x_{k}^{\prime}-a_{k}\right\|}+\left|\frac{\left\|x_{k}-a_{k}\right\|}{\left\|x_{k}^{\prime}-a_{k}\right\|}-1\right| .
\end{aligned}
$$

If $\left\|x_{k}-a_{k}\right\| \geq\left\|x_{k}^{\prime}-a_{k}\right\|$, then Eq. 111 holds true since

$$
\left|\frac{\left\|x_{k}-a_{k}\right\|}{\left\|x_{k}^{\prime}-a_{k}\right\|}-1\right|=\frac{\left\|x_{k}-a_{k}\right\|}{\left\|x_{k}^{\prime}-a_{k}\right\|}-1 \leq \frac{\left\|x_{k}-x_{k}^{\prime}\right\|+\left\|x_{k}^{\prime}-a_{k}\right\|}{\left\|x_{k}^{\prime}-a_{k}\right\|}-1=\frac{\left\|x_{k}-x_{k}^{\prime}\right\|}{\left\|x_{k}^{\prime}-a_{k}\right\|} .
$$

Otherwise, i.e., if $\left\|x_{k}-a_{k}\right\|<\left\|x_{k}^{\prime}-a_{k}\right\|$, Eq. 111 also holds true since

$$
\left|\frac{\left\|x_{k}-a_{k}\right\|}{\left\|x_{k}^{\prime}-a_{k}\right\|}-1\right|=1-\frac{\left\|x_{k}-a_{k}\right\|}{\left\|x_{k}^{\prime}-a_{k}\right\|} \leq 1-\frac{\left\|x_{k}^{\prime}-a_{k}\right\|-\left\|x_{k}-x_{k}^{\prime}\right\|}{\left\|x_{k}^{\prime}-a_{k}\right\|}=\frac{\left\|x_{k}-x_{k}^{\prime}\right\|}{\left\|x_{k}^{\prime}-a_{k}\right\|} .
$$

Hence Eq. 111 has been proved.

Finally, a combination of Eq. 110, Eq. 111 and Eq. 98 yields that

$$
\left\|\frac{x_{k}^{\prime}-a_{k}}{\left\|x_{k}^{\prime}-a_{k}\right\|}-\frac{x_{k}-a_{k}}{\left\|x_{k}-a_{k}\right\|}\right\| \leq 2 \frac{\left\|x_{k}-x_{k}^{\prime}\right\|}{\left\|x_{k}^{\prime}-a_{k}\right\|} \leq 2 \sqrt{\frac{2 \varepsilon^{\prime}-\varepsilon^{\prime 2}}{4-6 \varepsilon^{\prime}+3 \varepsilon^{\prime 2}}}<\varepsilon, \quad \forall k \geq N,
$$

which is Eq. 97 and hence the proof is complete.

We now have sufficient information for addressing Questions 5 and 6.

Remark 12 (answer to Question 5) In view of Theorem 4, the two cones $\bar{N}_{A, B}^{c}(\bar{x})$ and $\bar{N}_{A, B}(\bar{x})$ are equal when restricted to the cone $C$ given by Eq. 84 . Recall that elements of $C$ are sufficient for characterizing intrinsic transversality in view of Theorem 3 . Their equality outside of $C$ remains unknown.

Remark 13 (answer to Question 6) The combination of Eq. 82 and Eq. 86 yields that

$$
\bar{N}_{A, B}^{c}(\bar{x}) \cap C=\bar{N}_{A, B}^{w}(\bar{x}) \cap C=\bar{N}_{A, B}(\bar{x}) \cap C .
$$

In view of Theorem 3, this particularly implies that the three cones have equivalent roles in characterizing intrinsic transversality. 
Remark 14 Corresponding to $\operatorname{itr}_{w}[A, B](\bar{x})$, the cone $\bar{N}_{A, B}^{w}(\bar{x})$ would not be essential for the analysis in this section due to Eq. 82 and Eq. 112. This explains why we chose not to introduce its definition for the sake of brevity in terms of terminology. It occasionally comes up in the discussion only for the purpose of addressing Question 6.

Remark 15 Thanks to Eq. 80 and Eq. 81, Theorem 1(ii) in the Euclidean space setting can be deduced from Theorem 4 . However, Theorem 4 can not be deduced from Theorem 1(ii) since in general the equality $\operatorname{itr}[A, B](\bar{x})=\operatorname{itr}_{c}[A, B](\bar{x})$ does not imply $\bar{N}_{A, B}^{c}(\bar{x}) \cap$ $C=\bar{N}_{A, B}(\bar{x}) \cap C$. Instead, Theorem 4 complements Theorem 1(ii) and further clarifies the characterizations of intrinsic transversality in terms of relative limiting normals. It is worth noting that the result of Theorem 4 is also inspired by the importance of the cones themselves, see [32, page 123].

We conclude this paper with a list of characterizations of intrinsic transversality in the Euclidean space setting.

Proposition 11 (characterizations of intrinsic transversality in Euclidean spaces) Let $A, B$ be closed sets and $\bar{x} \in A \cap B$. The following conditions are equivalent:

(i) $\{A, B\}$ is intrinsically transversal at $\bar{x}$;

(ii) $\operatorname{itr}_{c}[A, B](\bar{x})>0$;

(iii) there exists a number $\alpha \in] 0,1\left[\right.$ such that $\left\|v_{1}+v_{2}\right\|>\alpha$ for all $\left(v_{1}, v_{2}\right) \in C \cap$ $\bar{N}_{A, B}^{c}(\bar{x}) \cap S$;

(iv) $\left\{v \in X \mid(v,-v) \in C \cap \bar{N}_{A, B}^{c}(\bar{x})\right\} \subset\{0\}$;

(v) there exists a number $\alpha \in] 0,1\left[\right.$ such that $\left\|v_{1}+v_{2}\right\|>\alpha$ for all $\left(v_{1}, v_{2}\right) \in$ $\bar{N}_{A, B}^{c}(\bar{x}) \cap S$;

(vi) $\left\{v \in X \mid(v,-v) \in \bar{N}_{A, B}^{c}(\bar{x})\right\} \subset\{0\}$;

(vii) $\operatorname{itr}[A, B](\bar{x})>0$;

(viii) there exists a number $\alpha \in] 0,1\left[\right.$ such that $\left\|v_{1}+v_{2}\right\|>\alpha$ for all $\left(v_{1}, v_{2}\right) \in C \cap$ $\bar{N}_{A, B}(\bar{x}) \cap S$;

(ix) $\left\{v \in X \mid(v,-v) \in C \cap \bar{N}_{A, B}(\bar{x})\right\} \subset\{0\}$;

(x) there exists a number $\alpha \in] 0,1\left[\right.$ such that $\left\|v_{1}+v_{2}\right\|>\alpha$ for all $\left(v_{1}, v_{2}\right) \in$ $\bar{N}_{A, B}(\bar{x}) \cap S$;

(xi) $\quad\left\{v \in X \mid(v,-v) \in \bar{N}_{A, B}(\bar{x})\right\} \subset\{0\}$;

(xii) $\operatorname{itr}_{w}[A, B](\bar{x})>0$;

(xiii) there exists a number $\alpha \in] 0,1\left[\right.$ such that $\left\|v_{1}+v_{2}\right\|>\alpha$ for all $\left(v_{1}, v_{2}\right) \in C \cap$ $\bar{N}_{A, B}^{w}(\bar{x}) \cap S$;

(xiv) $\left\{v \in X \mid(v,-v) \in C \cap \bar{N}_{A, B}^{w}(\bar{x})\right\} \subset\{0\}$;

(xv) there exists a number $\alpha \in] 0,1\left[\right.$ such that $\left\|v_{1}+v_{2}\right\|>\alpha$ for all $\left(v_{1}, v_{2}\right) \in$ $\bar{N}_{A, B}^{w}(\bar{x}) \cap S$;

(xvi) $\left\{v \in X \mid(v,-v) \in \bar{N}_{A, B}^{w}(\bar{x})\right\} \subset\{0\}$;

(xvii) $\operatorname{itr}_{p}[A, B](\bar{x})>0$;

(xviii) $\{A, B\}$ satisfies property $(\mathcal{P})$ at $\bar{x}$.

If, in addition, the sets are convex, then the following item can be added to the above list:

(xix) $\{A, B\}$ is subtransversal at $\bar{x}$. 
Proof (i) $\Leftrightarrow$ (ii) follows from Theorem 1. (i) $\Leftrightarrow$ (iii) $\Leftrightarrow$ (iv) follows from Theorem 3. (i) $\Leftrightarrow$ (v) $\Leftrightarrow$ (vi) follows from Corollary 3 of [32]. (i) $\Leftrightarrow$ (vii) follows from Proposition 3(iii). (i) $\Leftrightarrow$ (viii) $\Leftrightarrow$ (ix) follows from Theorem 3 and Theorem 4. (i) $\Leftrightarrow$ (x) $\Leftrightarrow$ (xi) follows from Theorem 5 of [32]. (i) $\Leftrightarrow$ (xii) $\Leftrightarrow$ (xiii) $\Leftrightarrow$ (xiv) $\Leftrightarrow$ (xv) $\Leftrightarrow$ (xvi) are consequences of the previous equivalences in view of Proposition 4(i) and the inclusions in Eq. 82. (i) $\Leftrightarrow$ (xvii) $\Leftrightarrow$ (xviii) follows from Theorem 2 and Proposition 5, respectively. (i) $\Leftrightarrow$ (xix) in the convex setting follows from Proposition 3(iv).

Remark 16 The equivalences (i) $\Leftrightarrow$ (ii) $\Leftrightarrow$ (v) $\Leftrightarrow$ (vi) $\Leftrightarrow$ (vii) $\Leftrightarrow$ (x) $\Leftrightarrow$ (xi) can be deduced from Theorem 3, Corollary 3 and Proposition 4 of [32]. Using Theorems 7 and 8 of the aforementioned paper, one can straightforwardly add more items to the above list of intrinsic transversality characterizations.

Acknowledgments NHT would like to thank the Centre for Informatics and Applied Optimization (CIAO) at Federation University Australia for offering him the opportunity for collaborating with his colleagues there in November 2018. Without that visit, this paper would not be completed. The authors would like to thank the two anonymous referees and Professor Alexander Kruger for their careful reading of the manuscript and constructive comments and valuable suggestions.

Open Access This article is licensed under a Creative Commons Attribution 4.0 International License, which permits use, sharing, adaptation, distribution and reproduction in any medium or format, as long as you give appropriate credit to the original author(s) and the source, provide a link to the Creative Commons licence, and indicate if changes were made. The images or other third party material in this article are included in the article's Creative Commons licence, unless indicated otherwise in a credit line to the material. If material is not included in the article's Creative Commons licence and your intended use is not permitted by statutory regulation or exceeds the permitted use, you will need to obtain permission directly from the copyright holder. To view a copy of this licence, visit http://creativecommons.org/licenses/by/4.0/.

\section{References}

1. Bakan, A., Deutsch, F., Li, W.: Strong CHIP, normality, and linear regularity of convex sets. Trans. Amer. Math. Soc. 357(10), 3831-3863 (2005)

2. Bauschke, H.H., Borwein, J.M.: On the convergence of von Neumann's alternating projection algorithm for two sets. Set-Valued Anal. 1(2), 185-212 (1993)

3. Bauschke, H.H., Borwein, J.M.: On projection algorithms for solving convex feasibility problems. SIAM Rev. 38(3), 367-426 (1996)

4. Bauschke, H.H., Borwein, J.M., Li, W.: Strong conical hull intersection property, bounded linear regularity, Jameson's property (G), and error bounds in convex optimization. Math. Program., Ser. A 86(1), 135-160 (1999)

5. Bauschke, H.H., Borwein, J.M., Tseng, P.: Bounded linear regularity, strong CHIP, and CHIP are distinct properties. J. Convex Anal. 7(2), 395-412 (2000)

6. Bauschke, H.H., Luke, D.R., Phan, H.M., Wang, X.: Restricted normal cones and the method of alternating projections: theory. Set-Valued Var. Anal. 21(3), 431-473 (2013)

7. Bivas, M., Krastanov, M., Ribarska, N.: On tangential transversality. J. Math. Anal. Appl. 481(1), 123455 (2020)

8. Bui, H.T., Kruger, A.Y.: Extremality, stationarity and generalized separation of collections of sets. J. Optim Theory Appl (2019)

9. Clarke, F.H.: Optimization and Nonsmooth Analysis. Wiley, New York (1983)

10. Dolecki, S.: Metrically upper semicontinuous multi]unctions and their intersections. Math. Res. Center, Madison Wis., Report 2035 (1980)

11. Dolecki, S.: Tangency and differentiation: Some applications of convergence theory. Ann. Mat. Pura Appl. 130(4), 223-255 (1982)

12. Dontchev, A.L., Rockafellar, R.T. Implicit Functions and Solution Mappings. A View from Variational Analysis, 2nd edn. Springer, New York (2014) 
13. Drusvyatskiy, D., Ioffe, A.D., Lewis, A.S.: Transversality and alternating projections for nonconvex sets. Found. Comput. Math. 15(6), 1637-1651 (2015)

14. Drusvyatskiy, D., Lewis, A.S.: Inexact alternating projections on nonconvex sets. arXiv:1811.01298, 1-15 (2018)

15. Guillemin, V., Pollack, A.: Differential Topology. Prentice-Hall, Inc., Englewood Cliffs (1974)

16. Hesse, R., Luke, D.R.: Nonconvex notions of regularity and convergence of fundamental algorithms for feasibility problems. SIAM J. Optim. 23(4), 2397-2419 (2013)

17. Hirsch, M.: Differential Topology. Springer, New York (1976)

18. Ioffe, A.D.: Approximate subdifferentials and applications. III. The metric theory. Mathematika 36(1), 1-38 (1989)

19. Ioffe, A.D.: Metric regularity and subdifferential calculus. Russian Math. Surveys 55, 501-558 (2000)

20. Ioffe, A.D.: Metric regularity - a survey. Part I. Theory. J. Aust. Math. Soc. 101(2), 188-243 (2016)

21. Ioffe, A.D.: Metric regularity - a survey. Part II. Applications. J. Aust. Math. Soc. 101(3), 376-417 (2016)

22. Ioffe, A.D.: Transversality in variational analysis. J. Optim. Theory Appl. 174(2), 343-366 (2017)

23. Ioffe, A.D.: Variational Analysis of Regular Mappings. Theory and Applications. Springer Monographs in Mathematics Springer (2017)

24. Khanh, P.Q., Kruger, A.Y., Thao, N.H.: On induction theorem and nonlinear regularity models. SIAM J. Optim. 25(4), 2561-2588 (2015)

25. Kruger, A.Y.: On Fréchet subdifferentials. J. Math. Sci. 116(3), 3325-3358 (2003)

26. Kruger, A.Y.: Stationarity and regularity of set systems. Pac. J. Optim. 1(1), 101-126 (2005)

27. Kruger, A.Y.: About regularity of collections of sets. Set-Valued Anal. 14(2), 187-206 (2006)

28. Kruger, A.Y.: About stationarity and regularity in variational analysis. Taiwanese J. Math. 13(6A), 17371785 (2009)

29. Kruger, A.Y.: Error bounds and metric subregularity. Optimization. 64(1), 49-79 (2015)

30. Kruger, A.Y.: Error bounds and Hölder metric subregularity. Set-Valued Var. Anal. 23(4), 705-736 (2015)

31. Kruger, A.Y.: Nonlinear metric subregularity. J. Optim. Theory Appl. 171(3), 820-855 (2016)

32. Kruger, A.Y.: About intrinsic transversality of pairs of sets. Set-Valued Var. Anal. 26(1), 111-142 (2018)

33. Kruger, A.Y., López, M.A.: Stationarity and regularity of infinite collections of sets. J. Optim. Theory Appl. 154(2), 339-369 (2012)

34. Kruger, A.Y., López, M.A.: Stationarity and regularity of infinite collections of sets. Applications to infinitely constrained optimization. J. Optim. Theory Appl. 155(2), 390-416 (2012)

35. Kruger, A.Y., Luke, D.R., Thao, N.H.: About subtransversality of collections of sets. Set-Valued Var Anal. 25(4), 701-729 (2017)

36. Kruger, A.Y., Luke, D.R., Thao, N.H.: Set regularities and feasibility problems. Math. Program., Ser. B 168(1), 1-33 (2018)

37. Kruger, A.Y., Thao, N.H.: About uniform regularity of collections of sets. Serdica Math. J. 39, 287-312 (2013)

38. Kruger, A.Y., Thao, N.H.: About $[q]$-regularity properties of collections of sets. J. Math. Anal. Appl. 416(2), 471-496 (2014)

39. Kruger, A.Y., Thao, N.H.: Quantitative characterizations of regularity properties of collections of sets. J. Optim. Theory Appl. 164(1), 41-67 (2015)

40. Kruger, A.Y., Thao, N.H.: Regularity of collections of sets and convergence of inexact alternating projections. J. Convex Anal. 23(3), 823-847 (2016)

41. Lewis, A.S., Luke, D.R., Malick, J.: Local linear convergence of alternating and averaged projections. Found. Comput. Math. 9(4), 485-513 (2009)

42. Lewis, A.S., Malick, J.: Alternating projections on manifolds. Math. Oper. Res. 33(1), 216-234 (2008)

43. Li, C., Ng, K.F., Pong, T.K.: The SECQ, linear regularity, and the strong CHIP for an infinite system of closed convex sets in normed linear spaces. SIAM J. Optim. 18(2), 643-665 (2007)

44. Luke, D.R., Teboulle, M., Thao, N.H.: Necessary conditions for linear convergence of iterated expansive, set-valued mappings. Math. Program. Ser. A, https://doi.org/10.1007/s10107-018-1343-8

45. Mordukhovich, B.S.: Variational Analysis and Generalized Differentiation, I: Basic Theory. Grundlehren der mathematischen Wissenschaften. Springer-Verlag, New York (2006)

46. Ng, K.F., Zang, R.: Linear regularity and $\varphi$-regularity of nonconvex sets. J. Math. Anal. Appl. 328, 257-280 (2007)

47. Ngai, H.V., Théra, M.: Metric inequality, subdifferential calculus and applications. Set-Valued Anal. 9(1-2), 187-216 (2001)

48. Noll, D., Rondepierre, A.: On local convergence of the method of alternating projections. Found. Comput. Math. 16(2), 425-455 (2016)

49. Penot, J.P.: Calculus without Derivatives. Graduate Texts in Mathematics. Springer, New York (2013) 
50. Phan, H.M.: Linear convergence of the Douglas-Rachford method for two closed sets. Optimization $\mathbf{6 5}$, 369-385 (2016)

51. Rockafellar, R.T., Wets, R.J.B.: Variational Analysis. Springer, Berlin (1998)

52. Thao, N.H.: A convergent relaxation of the Douglas-Rachford algorithm. Comput. Optim. Appl. 70(3), 841-863 (2018)

53. Ye, J.J., Ye, X.Y.: Necessary optimality conditions for optimization problems with variational inequality constraints. Math. Oper. Res. 22(4), 977-997 (1997)

54. Zheng, X.Y., Ng, K.F.: Linear regularity for a collection of subsmooth sets in Banach spaces. SIAM J. Optim. 19(1), 62-76 (2008)

Publisher's Note Springer Nature remains neutral with regard to jurisdictional claims in published maps and institutional affiliations.

\section{Affiliations}

\section{Nguyen Hieu Thao ${ }^{1,2}$ (D) $\cdot$ Hoa T. Bui ${ }^{3} \cdot$ Nguyen Duy Cuong $^{4,5} \cdot$ Michel Verhaegen $^{1}$}

Hoa T. Bui

hoa.bui@curtin.edu.au

Nguyen Duy Cuong

duynguyen@students.federation.edu.au; ndcuong@ctu.edu.vn

Michel Verhaegen

m.verhaegen@tudelft.nl

1 Delft Center for Systems and Control, Delft University of Technology, 2628CD Delft, The Netherlands

2 Department of Mathematics, Teacher College, Can Tho University, Can Tho City, Vietnam

3 School of Electrical Engineering, Computing, and Mathematical Sciences, Curtin University, Perth, WA, 6102, Australia

4 Centre for Informatics and Applied Optimization, Federation University Australia, POB 663, Ballarat, VIC 3350, Australia

5 Department of Mathematics, College of Natural Sciences, Can Tho University, Can Tho City, Vietnam 